Y. KAMETAN1

KODAI MATH. J.

16 (1993), $138-170$

\title{
TORUS SUM FORMULA OF SIMPLE INVARIANTS FOR 4-MANIFOLDS
}

\author{
BY YUKIO KAMETANI
}

\section{Introduction.}

The topology of moduli spaces of anti-self-dual (ASD) connections is closely related with differentiable structures on 4-manifolds. In his celebrated paper [6], Donaldson has defined the polynomial invariants to distinguish differentiable structures on a 4-manifold. Though a significant result on the vanishing has been obtained at the same time, these invariants remain to be very difficult to determine completely. In fact, many examples have been calculated using an identification of irreducible ASD connections and stable vector bundles by Donaldson ([6], [7], [9], [12], [18]). But there are another direct approaches in the case that the dimension of ASD moduli is zero and that the invariant is just a number of the points in the moduli. For example Gompf and Mrowka have defined an invariant for 4-manifolds with torus end, using 0 or 1 dimensional ASD moduli, and proved that the invariant of the glued manifolds with solid torus can be emerged as the number of ASD connections which can be extended to over the solid torus. From a topological argument on K3 surface with elliptic fibration, they calculated the above numbers for fake K3 surfaces obtained by performing logarithmic transformations on embedded 2-tori. After that, Kromheimer has observed that the ASD moduli of Kummer surface comes down to the flat moduli as all $(-2)$ curves tends to infinity, so the invariant could be computed algebraically [13]. The invariant obtained by 0 -dimensional ASD moduli is said to be a simple invariant.

In this paper we give a torus sum formula of simple invariants for 4-manifolds. Our idea and formula are simple. Suppose that we have two simply connected closed 4-manifolds which contain a 2-torus with the trivial normal bundle. We assume that the complements are simply connected and the second Stiefel-Whitney class to define the $S O(3)$ bundle does not vanish on the 2-torus. Then any ASD connection converges to some ASD connections as the 2-torus tends to infinity. On the other hand, any ASD connection over the new 4manifold obtained by torus sum also converges to some ASD connections as the bi-collar of the intermediate 3-torus is stretched to infinity. Hence we prove that the simple invariant of the new 4-manifold is the product of that of the

Received April 8, 1992 , Revised November, 25, 1992. 
given 4-manifolds. This formula is a variant of a relation of Donaldson invariants to Floer homology in Atiyah's exposition [1].

We can apply this formula to compute the simple invariant for regular elliptic surfaces. The rational elliptic surface has the least Euler number among them. The K3 surface is obtained by gluing two rational elliptic surfaces as fiber sum [17]. Gluing more rational elliptic surfaces yields all the other regular elliptic surfaces without multiple fibers. On the other hand, the simple invariant of the $\mathrm{K} 3$ surface has been known to be 1 for all second StiefelWhitney classes ([5], [13]). Hence the value is 1 for second Stiefel-Whitney classes whose restriction to the fiber are non-zero. In particular, if the geometric genus is even, then all the value is 1 .

We remark that these calculations improve two known facts the first, Sato and the author have shown that the value is non-zero for a second StiefelWhithey class, by using stable vector bundles [11]. The second, Ue has shown that the value of the above is independent of the choice of second StiefelWhitney class with the additional condition, by analyzing the action of the diffeomorphism groups on second cohomology groups [21].

I would like to thank Professor M. Furuta for valuable communications with me.

\section{Review of simple invariant and main result}

We recall the simple invariant $\gamma$ defined by Donaldson ([6], [7, Chapter 9]) let $X$ be an oriented closed smooth 4-manifold with the following properties;

(A1) $\pi_{1}(X)=1$

(A2) $b_{+}(X) \geqq 3$ and $b_{+}(X)$ is odd,

where $b_{+}$denotes the dimension of the maximal positive subspacee for the intersection on $H^{2}(X)$. Then there is a well defined lift of $\bmod 2$ cup square called the Pontrjagin square $H^{2}\left(X \boldsymbol{Z}_{2}\right) \rightarrow H^{4}\left(X ; \boldsymbol{Z}_{4}\right)$. Composing this map with evaluation on the fundamental class defines a quadratic map $H^{2}\left(X \boldsymbol{Z}_{2}\right) \rightarrow \boldsymbol{Z}_{4}$. Let $P \rightarrow X$ be the principal $S O(3)$ bundle with $w_{2}(P)=\eta$ and $p_{1}(P)=l$. They satisfy $\eta^{2} \equiv l(\bmod 4)$. A theorem of Dold and Whitney [4] tells us that $S O(3)$ bundles over a compact 4-manifold are determined completely by $\eta$ and /. Conversely, given $\eta$ and / with $\eta^{2} \equiv l(\bmod 4)$, we can easily construct the corresponding $S O(3)$ bundle over $X$. We give $X$ a Riemannian metric. Then the affine space $\mathcal{A}_{P}$ of $L_{3}^{2}$ connections has a natural Banach structure from the $L_{3}^{2}$ norm. The $L_{4}^{2}$ gauge group $\mathcal{G}_{P}$ is a Banach Lie group. The Lie algebra of $\mathcal{G}_{P}$ is $L_{4}^{2}(\operatorname{Ad} P)$. Let $A_{P}^{*} \subset \mathcal{A}_{P}$ denote the subset of irreducible connections. Then the quotient $\mathscr{B}_{P}^{*}=\mathcal{A}_{P}^{*} / \mathcal{G}_{P}$ is a $C^{\infty}$-Banach manifold such that the projection $\pi: \mathcal{A}_{P}^{*} \rightarrow \mathscr{B}_{P}^{*}$ defines a principal $\mathcal{G}_{P}$-bundle $([8],[7,4.2])$. The tangent space to $[A] \in \mathscr{B}_{P}^{*}$ is isomorphic to $\left\{a \in L_{3}^{2}\left(\Omega_{X}^{1}(\operatorname{Ad} P)\right) \mid d_{A}^{*} a=0\right\}$. We say an element $[A]$ in $\mathcal{B}_{P}^{*}$ to be regular if the operator $d_{A}^{+}$is surjective. Let $\mathscr{M}_{X}(l, \eta, g)$ denote the moduli space of $g$-ASD connections on $P$. Then the formal dimension is equal to 
$-2 l-3\left(1+b_{+}(X)\right)$ [2]. We choose $l_{X}$ so that $-2 l_{X}-3\left(1+b_{+}(X)\right)=0$. Let $\mathcal{C}_{X}^{r}$ $(r \geqq 3)$ denote the space of $C^{r}$-metrics on $X$. By ([8], [7, 4.3]), there is a Baire set $C^{\prime} \chi$ in $\mathcal{C}_{X}^{r}$ such that for all $g \in \mathcal{C}_{X}^{\prime}, \mathscr{M}_{X}(l x, \eta, g)$ is a finite set consisting of irreducible regular connections. We fix $g \in \mathcal{C}_{X}^{\prime}$. Then we can define a sign at any $[A] \in \mathscr{M}_{X}\left(l_{X}, \eta, g\right)$ using a line bundle over $\mathscr{B}_{P}^{*}$. For $A \in \mathcal{A}_{P}^{*}$, we consider the deformation complex

$$
\delta_{A}=d_{A}^{*} \oplus d_{A}^{+}: L_{3}^{2}\left(\Omega_{X}^{1}(\operatorname{AdP})\right) \longrightarrow L_{2}^{2}\left(\left(\Omega_{X}^{0} \oplus \Omega_{X}^{+}\right)(\operatorname{Ad} P)\right) .
$$

We choose a linear map $\mathrm{S}: \boldsymbol{R}^{N} \rightarrow L_{2}^{2}\left(\Omega_{X}^{+}(\operatorname{Ad} P)\right)$ so that $\delta_{A} \oplus S$ is surjective. Then we define the determinant line of $\delta_{A} \oplus S$ by

$$
\Lambda_{P, A}=\left(\Lambda^{\max } \operatorname{Ker}\left(\boldsymbol{\delta}_{A} \oplus S\right)\right) \otimes\left(\Lambda^{N} \boldsymbol{R}^{N}\right)^{*} .
$$

This line has an intrinsic sense by the exact sequence

$$
0 \longrightarrow \operatorname{Ker} \boldsymbol{\delta}_{A} \longrightarrow \operatorname{Ker}\left(\boldsymbol{\delta}_{A} \oplus S\right) \stackrel{\pi_{A}}{\rightarrow} \boldsymbol{R}^{N} \longrightarrow \operatorname{Coker} \boldsymbol{\delta}_{A} \longrightarrow 0 .
$$

Since the surjectivity holds in a neighborhood of $A$, these lines are patched together to get a locally trivial line bundle over $\mathcal{A}_{D}^{*}$. It descends to the determinant line bundle $\Lambda_{P} \rightarrow \mathscr{B}_{P}^{*}$ by the free action of $\mathcal{G}_{P}$. The bundle $\Lambda_{P} \rightarrow \mathscr{B}_{P}^{*}$ is topologically trivial $([5],[7,5.4])$. Since $\delta_{A}$ is an isomorphism for any $[A] \in$ $\mathscr{M}_{X}\left(l_{X}, \eta, g\right)$, we can define a section on $\Lambda_{P}$ at $[A]$ by

$$
\left(\pi_{A}^{-1}\left(e_{1}\right) \wedge \cdots \wedge \pi_{A}^{-1}\left(e_{N}\right)\right) \otimes\left(e_{1} \wedge \cdots \wedge e_{N}\right)^{*},
$$

where $e_{1}, \cdots, e_{N}$ form a basis of $\boldsymbol{R}^{N}$. This defines an orientation $o([A])$ of the the line bundle $\Lambda_{P} \rightarrow \mathscr{B}_{P}^{*}$. For a later use, we remark that this section is defined on a connected region $U([A])$ consisting of irreducible regular connections about $[A]$.

On the other hand, for an orientation $\Omega$ of $H^{+}(X)$ and an integral lift $c$ of $\eta$, there is another orientation $o(\Omega)$ determined at a connection obtained by attaching some standard instantons over $\mathrm{S}^{4}$ with reducible connection determined by $c([5],[7,7.1 .6])$. Then the sign $\varepsilon([A])$ at $[A] \in \mathscr{M}_{X}\left(l_{X}, \eta, g\right)$ is given by $o([A])=\varepsilon([A]) o(\Omega)$ and the simple invariant is defined by

$$
\gamma_{X}(\eta)=\sum_{[A] \in \mathscr{M}_{X}\left(l_{X}, \eta, g\right)} \varepsilon([A]) .
$$

This function $\gamma_{X}$ is independent of the choice of the element $g$ in $\mathcal{C}_{X}^{\prime}$ and satisfies the following ([6], [7]): If $\phi: X \rightarrow X^{\prime}$ is an orientation preserving diffeomorphism, then $\gamma_{X}\left(\phi^{*}\left(\eta^{\prime}\right)\right)=\varepsilon(\phi) \gamma_{X}\left(\eta^{\prime}\right)$ where $\varepsilon(\phi)$ is -1 if either $\phi^{*}$ is an orientation reversing map from $H^{+}\left(X^{\prime}\right)$ onto $H^{+}(X)$ or $\left(\left(c-\phi^{*}\left(c^{\prime}\right)\right) / 2\right)^{2} \equiv 1(\bmod 2)$ but not both and is 1 otherwise. (Here $c^{\prime}$ denotes the integral lift of $\eta^{\prime}$ and $c$ is the integral lift of $\phi^{*}\left(\eta^{\prime}\right)$ used in orientaring their respective moduli spaces.) Hence the absolute value $\left|\gamma_{\boldsymbol{x}}\right|$ can be thought of a function on

$$
C_{X}=\left\{\eta \in H^{2}\left(X ; \boldsymbol{Z}_{2}\right) \mid \eta \neq 0, \eta^{2} \equiv l_{X}(\bmod 4)\right\} .
$$


We return to our main theorem. Let $K$ be a compact oriented smooth 4-manifold with boundary $Z=T^{3}$, satisfying the following;

(B1) $\pi_{1}(K)=1$,

(B2) $\quad b_{+}(K) \geqq 2$ and $b_{+}(K)$ is even.

For two such manifolds $K_{1}, K_{2}$ and an orientation reversing diffeomorphism $\phi: \partial K_{1} \rightarrow \partial K_{2}$, the oriented 4-manifold $X=K_{1} \cup_{\psi} K_{2}$ always satisfies (A1), (A2). Let $\sigma: Z \rightarrow X, \sigma_{i}: K_{i} \rightarrow X(i=1,2)$ be the inclusion. For each $\eta \in C_{X}$ with $\sigma^{*}(\eta)$ $\neq 0$, we can define an orientation reversing diffeomorphism $\phi_{i}: \partial K_{i} \rightarrow Z$ such that $\phi_{i}^{*}(\eta)$ can be extended to a class $\eta_{i}^{*} \in H^{2}\left(K_{i}^{*} ; \boldsymbol{Z}_{2}\right)$. The oriented closed 4-manifold $K_{\imath}^{*}=K_{i} \cup_{\phi_{i}} W$ also satisfies (Al), (A2). Here $W$ is the solid torus $T^{2} \times D^{2}$.

THEOREM 2.1. // $\eta_{\imath}^{*}$ satisfies $\left(\eta_{\imath}^{*}\right)^{2} \equiv l_{K_{\imath}^{*}}(\bmod 4)$ for each $\imath=1,2$, then

$$
\left|\gamma_{X}(\eta)\right|=\left|\gamma_{K_{1}^{*}}^{*}\left(\eta_{1}^{*}\right)\right|\left|\gamma_{K_{2}^{*}}^{*}\left(\eta_{2}^{*}\right)\right| \text {. }
$$

THEOREM 2.2. // $\eta_{\imath}^{*}$ does not satisfy $\left(\eta_{\imath}^{*}\right)^{2} \equiv l_{K_{i}^{*}}(\bmod 2)$ for some $i=1,2$, then $\gamma_{X}(\eta)=0$.

Remarks. (1) Let P. D. be the mod 2 Poincaré dual. Then $\eta_{\imath}^{*}+$ P. D. $\left[T^{2} \times 0\right]$ satisfies $\left(\eta_{i}^{*}+\text { P. D. }\left[T^{2} \times 0\right]\right)^{2} \equiv\left(\eta_{i}^{*}\right)^{2}+2(\bmod 4)$ and by the exact sequence

$$
0 \longrightarrow H^{2}\left(W, Z \quad Z_{2}\right) \longrightarrow H^{2}\left(\begin{array}{lll}
K_{\imath}^{*} & Z_{2}
\end{array}\right) \stackrel{\sigma_{\imath}^{*}}{\longrightarrow} H^{2}\left(K_{\imath} Z_{2}\right),
$$

it is only another choice for $77^{*}$. So the conditions of Theorem 2.1 and 2.2 are complementary to each other.

(2) In our application, if $K_{i}^{*}$ and $\eta_{i}^{*}(i=1,2)$ are chosen, then we will write $X=K_{1}^{*} K_{2}^{*}$ and $\eta=t \wedge \eta \ddot{i}$.

\section{Setting up gauge theory}

We first argue the ASD moduli over a 4-manifold with torus end. According to Taubes [20], we study a gauge theory on a convenient subspace of connections to apply a known analysis and to contain all ASD connections by some gauge. The uniqueness of flat connections over the torus enable us to apply his argument directly. For $n=2,3$, we denote by $\chi\left(T^{n}\right)$ the set of the conjugacy classes of representations from $\pi_{1}\left(T^{n}\right)$ to $S O(3)$. The topology of $\chi\left(T^{n}\right)$ has been discussed in [10, Proposition V.2.1]. Given a representations $p$, we form the associated flat $\boldsymbol{R}^{3}$ bundle $\xi_{\rho}$. Then we define a map

$$
\omega_{2}: \chi\left(T^{n}\right) \rightarrow H^{2}\left(T^{n} \quad Z_{2}\right)
$$

by $\omega_{2}(\rho)=\omega_{2}\left(\xi_{\rho}\right)$. This is surjective. We denote by $\chi_{\alpha}\left(T^{n}\right)$ the preimage of $\alpha \in H^{2}\left(\begin{array}{ll}T^{n} & Z_{2}\end{array}\right)$. Then the decomposition

$$
\chi\left(T^{n}\right)=\cup_{\alpha \in H^{2}\left(T n: Z_{2}\right)} \chi_{\alpha}\left(T^{n}\right)
$$


decomposes $\chi\left(T^{n}\right)$ into connected components. $\chi_{0}\left(T^{n}\right)$ is homeomorphic to $T^{n} / \pm 1$ and the other 7 components are isolated points whose stabilizers are isomorphic to $\boldsymbol{Z}_{2} \times \boldsymbol{Z}_{2}$. Any representation $p$ in the isolated points is regular, that is $H^{i}\left(T^{n} ; \operatorname{Ad} \rho\right)=0(0 \leqq \imath \leqq 2)$.

For the unique non-zero element $\alpha \in H^{2}\left(W ; \boldsymbol{Z}_{2}\right)$, we let $\tilde{Q}, \tilde{\Gamma}$ and $\mathscr{R}$ be the corresponding flat bundle, flat connection and stabilizer respectively. We write $Q=\left.\tilde{Q}\right|_{z}$ and $\Gamma=\left.\tilde{\Gamma}\right|_{z}$ Then the stabilizer of $\Gamma$ is just $\mathcal{R}$.

Let $K$ denote a compact oriented smooth 4-manifold with boundary $\mathrm{Z}$, satisfying (B1) and (B2). Let $\sigma: Z \rightarrow K$ be the inclusion. Define $Y=K \cup Z \times$ $[0, \infty)$. We choose $\eta \in H^{2}\left(K ; \boldsymbol{Z}_{2}\right)$ with $\tau^{*}(\eta) \neq 0$. Let $P_{0}$ be the $S O(3)$ bundle over $K$ with $w_{2}\left(P_{0}\right)=\eta$ and $p_{1}\left(P_{0}\right)=0 \in H^{4}(K \boldsymbol{Z})=0$. For a bundle isomorphism ८: $\sigma^{*}\left(P_{0}\right) \rightarrow Q$, define $P=P_{0} \cup \pi^{*} Q$, where $\pi: \mathrm{Zx}[0, \infty) \rightarrow Z$ is the projection. We give $Y$ a metric which is the product metric $h+d t^{2}$ on $Z \times[0,00$ ), where $h$ is a metric on $Z$. Let $\tau: Y \rightarrow \boldsymbol{R}$ be a smooth function such that $\tau(x, t)=t$ on $\boldsymbol{Z} \times[1,00)$ and $\tau=0$ on $K$. We fix a smooth connection $A_{0}$ on $P$ such that $A_{0}$ is equal to $\pi^{*} \Gamma$ over $\mathrm{Z} \chi\left[0\right.$, oo). We write $\left(-1 / 4 \pi^{2}\right) \int_{Y} \operatorname{Tr}\left(F_{A_{0}} \wedge F_{A_{0}}\right)=l(\in \boldsymbol{Z})$. For $p \geqq 1, k \geqq 0$ and $\delta>0$, we define the weighted norm $L_{k, \delta}^{p}(Y)$ by

$$
\|s\|_{L_{k, \delta}^{p}(Y)}=\left(\int_{Y} e^{\tau \delta} \sum_{l=0}^{k}\left|\nabla_{A_{0}}^{(l)} s\right|^{p}\right)^{1 / p} .
$$

Then we consider the following class of connections and gauge group on $P$

$$
\begin{aligned}
& \mathcal{A}_{P}=\left\{A_{0}+a \mid a \in L_{3,10 \mathrm{loc}}^{2}\left(\Omega_{Y}^{1}(\operatorname{Ad} P)\right),\|a\|_{L_{3, \delta}^{2}(Y)}<\infty\right\}, \\
& \mathcal{G}_{P}=\left\{u \in L_{4,10 c}^{2} \text { (AutP) I }\left\|\nabla_{A_{0}} u\right\|_{L_{3, \delta}^{2}(Y)}<\infty\right\}
\end{aligned}
$$

By the argument in [20, Section 7], we see that there is a well defined map $r: \mathcal{G}_{P} \rightarrow \mathscr{R}$ given by

$$
r(u)=\lim _{t \rightarrow \infty} u_{t}
$$

where the limit is in $C^{0}$-convergence. We note that the automorphisms in $\mathcal{G}_{P}$ are continuous by the Sobolev embedding theorem $L_{3,10 c}^{2}-\rightarrow C^{0}$.

LEMMA 3.1. The automorphism $a \in \mathscr{R}$ over $d K$ can be extended to all over $K$ continuously if and only if $a=1$.

Proof. The primary difference $\mathfrak{D}\left(\left.1\right|_{\partial K}, a\right)[19, \S 36]$ between the identity $1 \in C^{0}$ (Aut $P_{0}$ ) and $a \neq 1$ is a non-zero element in $H^{1}\left(\partial K ; Z_{2}\right)$. If $a$ can be extended to $K$, then by the extension theorem [19,37.11], there is an element $v$

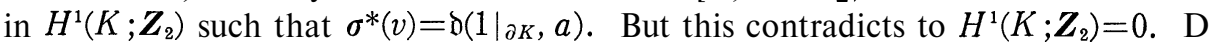

COROLLARY 3.2. The image of the map $r$ is $\{1\}$ and there are no reducible connections in $\mathscr{B}_{P}$. 
A gauge theory on 4-manifolds with cylindrical end has been studied by Taubes [20, Section 7]. Using his argument, we can prove the following two lemmas (see also [16]). We topologize $\mathcal{A}_{P}, \mathcal{G}_{P}$ by the norm $\|\cdot\|_{L_{3, \delta}^{2}}, \|\left.\nabla_{A_{0}}\right|_{L_{3, \delta}^{2}}$ respectively. Then

LEMM 3.3. $\mathcal{G}_{P}$ is a Banach Lie group. The Lie algebra of $\mathscr{G}_{P}$ is

$$
\mathrm{g}_{P}=\left\{\sigma \in L_{4,10 \mathrm{c}}^{2}(\operatorname{Ad} P) \mid\left\|\nabla_{A_{0}} \sigma\right\|_{L_{3, \delta}^{2}(Y)}<\infty\right\} .
$$

$\mathcal{G}_{P}$ acts smoothly on $\mathcal{A}_{P}$. The set $\left\{u \in L_{4,10 \mathrm{c}}^{2}(\right.$ Aut $\left.P) u \mathcal{A}_{P}=\mathcal{A}_{P}\right)$ is $\mathcal{G}_{P}$.

LEMMA 3.4. The quotient $\mathscr{B}_{P}=\mathcal{A}_{P} / \mathscr{G}_{P}$ is a $C^{\infty}$-Banach manifold such that the projection $\mathcal{A}_{P} \rightarrow \mathscr{B}_{P}$ defines a principal $\mathcal{G}_{P}$-bundle. The tangent space to $[A]$ $\in \mathscr{B}_{P}$ is isomorphic to

$$
\left\{a \in L_{3,10 \mathrm{c}}^{2}\left(\Omega_{Y}^{1}(\operatorname{Ad} P)\right) \mid\|a\|_{L_{3, \delta^{(Y)}}^{2}}<\infty, e^{-\tau \delta} d_{A}^{*} e^{\tau \delta} a=0\right\} .
$$

LEMMA 3.5. For bundle isomorphisms $c, \iota^{\prime}: \sigma_{*}\left(P_{0}\right) \rightarrow Q$, we consider $S O(3)$ bundles $P=P_{0} \cup_{\imath} \pi^{*} Q, P^{\prime}=P_{0} \cup_{\imath} \pi^{*} Q$, and $C^{m}(m \in N)$-connections $A_{0}, A_{0}^{\prime}$ on $P, P^{\prime}$ such that $A_{0}, A_{0}^{\prime}$ are equal to $\pi^{*} \Gamma$ over $Z \times[0, \infty)$ respectively. Then the following two conditions are equivalent.

(1) The automorphism $\left(\iota^{\prime}\right)^{-1}$ re over $d K$ can be extended to all over of $K$ continuously for some $r \in \mathscr{R}$ with $\mathrm{b}\left(1,\left(\iota^{\prime}\right)^{-1} r \iota\right)=0$.

(2) $\left(-1 / 4 \pi^{2}\right) \int_{Y} \operatorname{Tr}\left(F_{A_{0}} \wedge F_{A_{0}}\right)=\left(-1 / 4 \pi^{2}\right) \int_{Y} \operatorname{Tr}\left(F_{A_{0}^{\prime}} \wedge F_{A_{0}^{\prime}}\right)$.

Proof. We consider $S O(3)$ bundles $P^{*}=P_{0} \cup, \widetilde{Q},\left(P^{\prime}\right)^{*}=P_{0} \cup_{\iota}, \widetilde{Q}$ over $K \cup W$ and $C^{m}$-connections $A^{*},\left(A^{\prime}\right)^{*}$ extended by $\tilde{\Gamma}$ respectively. If the condition (1) holds, then the connections $A_{0}$ and $A_{0}^{\prime}$ have the same integral by Chern-Weil formula. Conversely if the condition (2) holds, then obviously $p_{1}\left(P^{*}\right)=p_{1}\left(\left(P^{\prime}\right)^{*}\right)$ and, moreover, $w_{2}\left(P^{*}\right)=w_{2}\left(\left(P^{\prime}\right)^{*}\right)$ for we can write $w_{2}\left(P^{*}\right)=w_{2}\left(\left(P^{\prime}\right)^{*}\right)+$ P.D. $\left[T^{2} \times 0\right]$, which induces $p_{1}\left(P^{*}\right)=p_{1}\left(\left(P^{\prime}\right)^{*}\right)+2(\bmod 4)$, a contradiction. By Dold-Whitney theorem, there exist bundle isomorphisms $f: P_{0} \rightarrow P_{0}$ and $h: \tilde{Q}_{Q} \rightarrow$ with $\iota^{\prime} f=h$ c. We prove that $\mathrm{A}$ is homotopic to some $r \in \mathscr{R}$. It suffices to prove that $\mathrm{A}_{T^{2}}$ is homotopic to some $r \in \mathscr{R}$. We remove an open 2-disk $D^{2}$ in $T^{2}$. By the homotopy classification theorem $[19,37.12]$, the assignment of the primary difference $\mathrm{b}(1, \cdot)$ in $H^{1}\left(T^{2} \backslash D^{2} ; \boldsymbol{Z}_{2}=H^{1}\left(T^{2} ; \boldsymbol{Z}_{2}\right) \cong \mathscr{R}\right.$ to each homotopy class in $\left[T^{2} \backslash D^{2}\right.$; Aut $Q]$ sets up a 1-1 correspondence between each sets. So $\left.\mathrm{A}\right|_{T^{2} \backslash D^{2}}$ is homotopic to some $r \in \mathscr{R}$ by a homotopy $H_{t}(0 \leqq t \leqq 1)$. We extend $H_{t}$ to all over to $T^{2}$, using the collar of $\partial D^{2}$. Since $\left[\left(D^{2}, \partial D^{2}\right) \quad(S O(3), \mathrm{id})\right]=\pi_{2}(S O(3))=1,\left.H_{1}\right|_{D^{2}}$ is homotopic to $r$ relative to $\partial D^{2}$, and so $\left(\iota^{\prime}\right)^{-1} r \iota$ is homotopic to $/$. We see that $b\left(1,\left(\iota^{\prime}\right)^{-1} r \iota\right)$ is zero by the argument in Lemma 3.1. D

COROLLARY 3.6. $\mathscr{B}_{P}$ depends only on $\eta$ and /. 
We return to the moduli of ASD connections. By [15, Theorem 1.1], there exists $\bar{\delta}>0$ such that for $0<\delta<\bar{\delta}, p \geqq 2$ and $k \geqq 0$, if $A$ is an ASD connection on $\mathrm{P}$, then the AHS complex

$$
0 \longrightarrow \Omega_{Y}^{0}(\mathrm{AdP}) \stackrel{d_{A}}{\longrightarrow} \Omega_{Y}(\mathrm{AdP}) \stackrel{d_{A}^{+}}{\longrightarrow} \Omega_{Y}^{+}(\mathrm{Ad} \mathrm{P}) \longrightarrow 0
$$

defines a Fredholm complex

$$
0 \longrightarrow L_{k+2, \delta}^{p}(Y) \stackrel{d_{A}}{\longrightarrow} L_{k+1, \delta}^{p}(Y) \stackrel{d_{A}^{+}}{\rightarrow} L_{k, \delta}^{p}(Y) \longrightarrow 0 .
$$

We denote by $H_{A}^{\imath}(0 \leqq i \leqq 2)$ the cohomology of the above complex. Then its index is given by [10, VI. 3]

$$
-\operatorname{dim} H_{A}^{0}+\operatorname{dim} H_{A}^{1}-\operatorname{dim} H_{A}^{2}=-2 l-3\left(2+b_{+}(Y)\right) .
$$

Let $\mathcal{C}_{Y}^{r}(r \geqq 3)$ be the space of all conformal classes of $C^{r}$-metrics on $Y$ which a Ie fixed metric $h+d t^{2}$ on $Z \times[0, \infty)$. If we fix a metric $\left[g_{0}\right] \in \mathcal{C}_{Y}^{r}$, then $\mathcal{C}_{Y}^{r}$ is identified with

$$
\left\{m: \Lambda^{+} \rightarrow \Lambda^{-} \quad C^{r} \text {-bundle map, sup }|m|<1,\left.m\right|_{Y \backslash K 0}=0\right\},
$$

where $\Lambda^{ \pm}$is \pm self-dual space with respect to $g_{0}$ and $K^{0}$ is the interior of $K$ $([7,1.1 .5])$. We choose $l_{Y}$ with $-2 l_{Y}-3\left(2+b_{+}(Y)\right)=0$.

PROPOSITION 3.7. There is a Baire set $\mathcal{C}_{Y}^{\prime} \subset \mathcal{C}_{Y}^{r}$ such that for all $l_{Y} \leqq l<0$ and $g \in \mathcal{C}_{Y}^{\prime}$, the $A S D$ moduli

$$
\mathscr{M}_{Y}(l, \eta, g)=\left\{[A] \in \mathscr{B}_{P} \mid F_{A}=-*_{g} F_{A}, \frac{-1}{4 \pi^{2}} \int_{Y} \operatorname{Tr}\left(F_{A} \wedge F_{A}\right)=l\right\}
$$

is a finite set consisting of irreducible regular connections. Any element in $\mathscr{M}_{Y}(l, \eta, g)$ has a smooth representative. Its dimension is equal to $-2 l-3\left(2+b_{+}(Y)\right)$. In particular if $l_{Y}<l<0$, then $\mathscr{M}_{Y}(l, \eta, g)$ is empty. Here the regularity at $[A]$ $\in \mathscr{M}_{Y}(l, \eta, g)$ means that $H_{A}^{2}=0$.

Proof. The regularity follows from the same argument as in [7, 4.3] (see also [14, 5 (iii)]). By the argument in [7, 4.2.3], we see that the representative in Coulomb gauge relative to some nearby smooth gauge is also smooth. We will prove the compactness in Appendix 2.

Remark. The proposition above has been stated in [10, Theorem V.3.3]. But we do not know how they orient the ASD moduli $\mathscr{M}_{Y}\left(l_{Y}, \eta, g\right)$ in spite of the non-existence of reducible connections in $\mathscr{B}_{P}$.

Let $\eta \in C_{X}, \eta_{1}^{*} \in C_{K_{1}^{*}}^{*}$ and $\eta_{2}^{*} \in C_{K_{2}^{*}}^{*}$ satisfy the assumption of Theorem 2.1. Let $\mathrm{P}^{*}$ be the $S O(3)$ bundle over $X$ with $w_{2}\left(P^{*}\right)=\eta$ and $p_{1}\left(P_{1}^{*}\right)=l_{K_{1}^{*}}$, and let $\mathrm{P}^{*}$ be the $S O(3)$ bundle over $K_{1}^{*}$ with $w_{2}\left(P_{1}^{*}\right)=\eta$ and $p_{1}\left(P_{1}^{*}\right)=l_{K_{1}^{*}}$. We write $P_{1}^{*}=P_{01} \bigcup_{\iota_{1}} Q$ for some bundle isomorphism $\iota_{1}:\left.P_{01}\right|_{z} \rightarrow Q$ and write $P^{*}=P_{01} \cup_{\iota_{2}^{-1} \iota_{1}} P_{02}$ 
for some bundle isomorphism $\iota_{2}: P_{02} \mid z_{\boldsymbol{z}} \rightarrow Q$. We put $P_{2}^{*}=P_{02} \cup_{\iota_{2}} \tilde{Q}$. We fix a smooth connection $A t$ on $P_{i}^{*}$ which is $f$ on $Q^{\sim}(i=1,2)$, and a smooth connection $A^{*}$ on $P$ which is $A_{\imath}^{*}$ on $P_{02}$. Then

$$
\begin{aligned}
p_{1}\left(P_{2}^{*}\right) & =\frac{-1}{4 \pi^{2}} \int_{K_{2}} \operatorname{Tr}\left(F_{A_{2}^{*}}^{*} \wedge F_{A_{2}^{*}}\right)=\frac{-1}{4 \pi^{2}} \int_{X} \operatorname{Tr}\left(F_{A} \wedge F_{A}\right)-\frac{-1}{4 \pi^{2}} \int_{K_{1}} \operatorname{Tr}\left(F_{A_{1}^{*}}^{*} \wedge F_{A_{2}^{*}}\right) \\
& =l_{X}-l_{K_{1}^{*}}=l_{K_{2}^{*}},
\end{aligned}
$$

and $w_{2}\left(P_{2}^{*}\right)=\eta_{2}^{*} \quad$ If not, then it must be $w_{2}\left(P_{2}^{*}\right)=\eta_{2}^{*}+P . D .\left[T^{2} \times 0\right]$, which induces $l_{K_{0}^{*}}^{*} \equiv l_{K_{0}^{*}}^{*}+2(\bmod 4)$. This is a contradiction. We define $Y_{2}=K_{i} \cup Z \times$ $[0, \infty)$ and $P_{i}=P_{0 i} \cup_{{ }_{i}} \pi^{*} Q$ for each $i=1,2$. We fix a connection $A_{02}$ on $P_{\imath}$ by

$$
A_{0 i}= \begin{cases}A^{*} & \text { over } K_{\imath}, \\ \pi^{*} \Gamma & \text { over } Z \times[0, \infty) .\end{cases}
$$

Using $A_{02}$ and $0<\delta_{0}<\delta$, we define the space $\mathcal{A}_{\boldsymbol{P}_{\boldsymbol{i}}}$, the gauge group $\mathcal{G}_{\boldsymbol{P}_{\boldsymbol{\imath}}}$ and the quotient $\mathscr{B}_{P_{i}}$.

We move on the gauge theory on 4-closed manifolds. We choose a lift $\ddot{\psi}: Q \rightarrow Q$ of $\phi: \partial K_{1} \rightarrow \partial K_{2}$ so that $\tilde{\psi} \Gamma=\Gamma$. For $n \in N$, let $X_{n}$ be the oriented closed 4-manifold defined by

$$
X_{n}=K_{1} \cup Z \times[0, n+1] \cup Z \times[0, n+1] \cup K_{2},
$$

where $\psi_{n}$ is the diffeomorphism on $Z \times[n-1, n+1]$ defined by using $\varphi$ and the reflection on $[n-1, n+1]$. Let $P_{n}$ be the $S O(3)$ bundle over $X_{n}$ defined by

$$
P_{n}=P_{01} \bigcup_{\iota_{1}} Q \times[0, n+1] \bigcup_{\widetilde{\psi}_{n}} Q \times[0, n+1] \bigcup_{\iota_{2}} P_{02},
$$

where $\tilde{\psi}_{n}$ is the automorphism on $Q \times[n-1, n+1]$ defined by using $\varphi$ and the reflection on $[n-1, n+1]$. We put

$$
\hat{A}_{0 n}= \begin{cases}A_{01} & \text { over } K_{1} \cup Z \times[0, n+1], \\ A_{02} & \text { over } K_{2} \cup Z \times[0, n+1] .\end{cases}
$$

Let $\mathcal{C}_{X_{n}}^{r}(r \geqq 3)$ be the space of all conformal classes of $C^{r}$-metrics on $X_{n}$ which are the fixed metric $h+d t^{2}$ on $Z \times[0, \mathrm{n}+1] \cup_{\psi_{n}} Z \times[0, n+1]$. If we fix a metric $\left[g_{0}\right]$ in $\mathcal{C}_{X_{n}}^{r}$, then $\mathcal{C}_{X_{n}}^{r}$ is identified with

$$
\left\{m: \Lambda^{+} \rightarrow \Lambda^{-} ; C^{r} \text {-bundle map, sup }|m|<1,\left.m\right|_{X_{n} \backslash\left(K_{1}^{0} \mathbb{I} K_{2}^{0}\right)}=0\right\},
$$

where $\Lambda^{ \pm}$is \pm self-dual space with respect to $g_{0}$. Then we have the following theorem, whose proof is also the same as in ([7, 4.3], $[14,5$ (iii) $]$ ).

PROPOSITION 3.8. There is a Baire set $\mathcal{C}_{X_{n}}^{\prime} \subset \mathcal{C}_{X_{n}}^{r}$ such that for all $g \in \mathcal{C}_{X_{n}}^{\prime}$, the ASD moduli $\mathcal{M}_{X_{n}}\left(l_{X_{n}}, \eta, g\right)$ is a finite set consisting of irreducible regular connections. 
For each $n \in N$, we fix a metric $g_{n}$ in $\mathcal{C}_{X_{n}}^{\prime}$ such that $g_{n}$ is independent of $n$ on $K_{2}^{0} \amalg K_{2}^{0}$ and the metric

$$
g_{i}= \begin{cases}g_{n} & \text { on } K_{\imath}, \\ h+d t^{2} & \text { on } Z \times[0, \infty),\end{cases}
$$

lies in $\mathcal{C}_{Y_{2}}^{\prime}$ for $\imath=1,2$. We can find these metric tenUejv, because the intersection of countable Baire sets is again a Baire set. For $n \in N^{\prime}$ we fix a function $\tau_{n}: X_{n} \rightarrow \boldsymbol{R}$ by

$$
\tau_{n}= \begin{cases}\tau_{1} & \text { on } \tau_{1}^{-1}([0, n-\varepsilon]), \\ \tau_{2} & \text { on } \tau_{2}^{-1}([0, n-\varepsilon]),\end{cases}
$$

to a small $0<\varepsilon<1$. We use the weighted norm $L_{k, \partial}^{p}\left(X_{n}\right)$ defined by

$$
\|s\|_{L_{k, \delta}^{p}\left(X_{n}\right)}=\left(\int_{X_{n}} e^{\tau} n^{\delta} \sum_{l=0}^{k}\left|\nabla_{A_{0}}^{(l)} s\right|^{p}\right)^{1 / p} .
$$

We note that if $\mathrm{s}$ is supported in $\tau_{2}^{-1}([0, n+1])$, then $\|s\|_{L_{k, \delta}^{p}\left(X_{n}\right)} \leqq\|s\|_{L_{k, \delta}^{p}\left(Y_{i}\right)}$ $\leqq e^{2 \delta}\|s\|_{L_{k, \delta}^{p}\left(X_{n}\right)}$, and if the support of $\mathrm{s}$ is contained in $\tau_{\imath}^{-1}([0, \mathrm{n}-1])$, then $\|s\|_{L_{k, \delta}^{p}\left(X_{n}\right)}=\|s\|_{L_{k, \delta}^{p}\left(Y_{i}\right)}$. We fix $p>4, q>8$ and $0<\delta_{0}, \delta<\bar{\delta}$ by

$$
\frac{1}{q}+\frac{1}{q}=\frac{1}{p}, \quad 0<\frac{\delta_{0}}{2}<\frac{\delta}{p}<\frac{\lambda}{2},
$$

where $\lambda^{2}$ is the first eigenvalue of $\Delta_{\Gamma}$ on $\operatorname{Ker} d_{\Gamma}^{*} \subset \Omega_{Z}^{1}(\operatorname{Ad} Q)$. The second inequality implies that $L_{3, \delta_{0}}^{2}\left(Y_{\imath}\right) \subset L_{1, \delta}^{p}\left(Y_{\imath}\right)[15$, Lemma 7.2].

\section{Decay estimate.}

In [22], Uhlenbech has proved that the curvature of ASD connections controls the uniform norm of the connection matrices in Coulomb gauge. Taubes has extended the result to ASD connections on the trivial bundle over 4-manifolds ([20] see also [3, Appendix A]). We show that his argument is applicable to ASD connections on flat bundles with an unique flat connection in the same way.

LEMMA 4.1. Let $U$ be an oriented open noncompact Riemannian 4-manifold. Let $U^{\prime} \Subset U$ be an interior domain with compact closure $\overline{U^{\prime}}$. We let $Q_{0} \rightarrow U$ be a flat $S O(3)$ bundle and $\Gamma_{0}$ be a canonical flat connection. Suppose that $\left.Q_{0}\right|_{\bar{U}^{\prime}} \rightarrow \bar{U}^{\prime}$ cannot admit any other flat connection topologically. Then there are constants $\varepsilon>0$ and $\zeta_{m, U^{\prime}}>0$ which depend on $U^{\prime}$ and $m \in N$ with the following significance: Let $A$ be an ASD connection on $P$ with $\left.\uparrow_{J U} F_{A}\right|^{2}<\varepsilon$. Then there exists $h \in$ $C^{\infty}\left(\right.$ Aut $\left.\left.Q_{0}\right|_{U^{\prime}}\right)$ such that 


$$
\sup _{U^{\prime}}\left\{\sum_{l=0}^{m}\left|\nabla_{\Gamma_{0}}^{(l)}\left(h A-\Gamma_{0}\right)\right|^{2}\right\} \leqq \zeta_{m, U^{\prime}} \int_{U}\left|F_{A}\right|^{2}
$$

Proof. We fix a locally finite open cover of $U$ by geodesic balls $\left\{B_{i}\right\}_{i \in N}$ such that the small balls $\left\{\widetilde{B}_{i}\right\}_{i \in N}$ with $1 / 2$ radius cover $U$ and the metric on $B_{2}$ is close to the Eucledian metric. Then by ([22], [7, Proposition (2.3.7), Theorem (2.3.8)]), there exists $\left\{h_{i} \in C^{\infty}\left(\operatorname{Iso}\left(\left.Q_{0}\right|_{B_{i}}, B_{\imath} \times S O(3)\right)\right)\right\}_{i \in N}$ such that $a_{\imath}=h_{\imath} A$ obeys $d^{*} a_{\imath}=0$ and

$$
\sup _{B_{i}^{\prime}}\left\{\sum_{\imath=0}^{m}\left|\nabla^{(l)} a_{\imath}\right|^{2}\right\} \leqq \zeta_{\imath} \int_{B_{\imath}}\left|F_{A}\right|^{2} .
$$

Here $B_{\imath}^{\prime}$ is the ball of radius $3 / 4$ (radius $\left(B_{\imath}\right)$ ) and $\nabla$ is the covariant derivative defined by the product structure. We denote by $\left\{\left(B_{i}^{\prime} \cap B_{j}^{\prime}\right)_{k}\right\}$ the connected components of $B_{i}^{\prime} \cap B_{\jmath}^{\prime}$. To obtain a desired gauge, we modify $h_{\imath}$, inductively. First we put $h_{1}^{\prime}=h_{1}$. Suppose that for $\jmath<i$ we defined $h_{j}^{\prime} \in C^{\infty}$ (Iso $\left(\left.Q_{0}\right|_{B_{j}}, B, \times\right.$ $S O(3)))$ such that $a_{\jmath}^{\prime}=h_{\jmath}^{\prime} A$ obeys

$$
\sup _{B_{j}^{\prime}}\left\{\sum_{i=n}^{m}\left|\nabla^{(l)} a_{j}^{\prime}\right|^{2}\right\} \leqq\left.\zeta_{j}^{\prime}\right|_{R_{j}}\left|F_{A}\right|^{2}
$$

for some modified constant $\zeta_{2}^{\prime}>0$. On $B_{i}^{\prime} \cap B_{\jmath}^{\prime}, h_{\imath j}^{\prime}=h_{i}\left(h_{j}^{\prime}\right)^{-1} \in C^{\infty}\left(B_{i}^{\prime} \cap B_{j}^{\prime} \quad S O(3)\right)$ obeys

$$
d h_{\imath \jmath}^{\prime}=h_{\imath j}^{\prime} a_{j}^{\prime}-a_{i} h_{\imath \jmath}^{\prime} \text {. }
$$

By bootstrapping, we see that $d h_{\imath \jmath}^{\prime}$ has the same $C^{m}$-bound as (4.1). If we choose $\varepsilon>0$ so small, we can write $h_{i j}^{\prime}=\exp \left(\xi_{i j k}^{\prime}\right) z_{\imath j k}^{\prime}$ for some constant $z_{i j k}^{\prime} \in$ 50(3) on $\left(B_{i}^{\prime} \cap B_{\imath}^{\prime}\right)_{k}$. We define $h_{i}^{\prime} \in C^{\infty}\left(\operatorname{Iso}\left(Q_{0} B_{i}, B_{\imath} \times S O(3)\right)\right)$ by

$$
h_{\imath}^{\prime}= \begin{cases}\exp \left(-\phi_{i j k} \xi_{i j k}^{\prime}\right) h_{\imath} & \text { on }\left(B_{i}^{\prime} \cap B_{i}^{\prime}\right)_{k}, \\ h_{\imath} & \text { on } B_{\imath}^{\prime} \backslash B_{\jmath}^{\prime},\end{cases}
$$

where $\phi_{i j k}$ is a cut-off function equal to 1 on $\left(\tilde{B}_{i} \cap \widetilde{B}_{j}\right)_{k}$ and is supported in $\left(B_{i}^{\prime} \cap B_{j}^{\prime}\right)_{k}$. Then $a_{\imath}^{\prime}=h_{\imath}^{\prime} A$ obeys the estimate (4.2) for a modified constant $\zeta_{i}^{\prime}>0$. In the construction above, we also obtained that $h_{i}^{\prime}\left(h_{j}^{\prime}\right)^{-1}=z_{\imath j}^{\prime}$ 冈n $\left(\widetilde{B}_{i} \cap \widetilde{B}_{j}\right)_{k}$ The data $\left\{\hat{B}_{i} \cap \bar{U}^{\prime}, z_{i j k}^{\prime}\right\}$ define a flat bundle $Q_{0}^{\prime} \rightarrow \bar{U}^{\prime}$ and a flat connection $\Gamma_{0}^{\prime}$ on $Q_{0}^{\prime}$. The data $\left\{\widetilde{B}_{i} \cap \bar{U}^{\prime},\left.h_{\imath}^{\prime}\right|_{\widetilde{B} \cap \bar{U}^{\prime}}\right\}$ define an isomorphism $h^{\prime}:\left.Q_{0}\right|_{\bar{U}} \rightarrow Q_{0}^{\prime}$. By the assumption, there is an isomorphism $h^{\prime \prime}:\left.Q_{0}^{\prime} \rightarrow Q_{0}\right|_{\bar{U}}$ such that $h^{\prime \prime} \Gamma_{0}^{\prime}=\Gamma_{0}$. (4.2) guarantees that $h-h^{\prime \prime} h^{\prime}$ obeys the desired estimate.

PROPOSITION 4.2. There are constants $\varepsilon>0$ and $\zeta=\zeta_{m}>0$, independent of $8 \leqq T \leqq \infty$, with the following signıficance Let $A$ be an ASD connection on $Q \times$ $(0, T)$ uith $\left.\int_{J Z \times(0, T)} F_{A}\right|^{2}<\varepsilon$, then there exists $h \in C^{\infty}(\operatorname{Aut}(Q \times(1, T-1)))$ suchthat for $8 \leqq t \leqq T-8$, 


$$
\sup _{Z \times\left[t-1, t^{+}+1\right](}\left(\sum_{l=0}^{m}\left|\nabla \pi^{(l)}\left(h A-\pi^{*} \Gamma\right)\right|_{\mathrm{J}}^{\prime} \leqq \zeta_{\mathrm{J} Z \times[t-8, t+8]}^{r}\left|F_{A}\right|^{2} .\right.
$$

Proof. The proof is essentially the same as that of [20, Lemma 10.5]. We apply Lemma 4.1 with $U=Z \times(j-1, j+8)$ and $U^{\prime}=Z \times[j, \jmath+7]$ for each $j \in 4 N$. Then there exists $h_{j} \in C^{\infty}(\operatorname{Aut}(Q \times[j, j+7]))$ such that

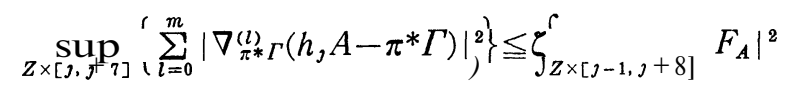

On $Z \times[j+4, j+7], h_{j, j+4}=h_{j} h_{j+4}^{-1} \in C^{\infty}(\operatorname{Aut}(Q \times[j+4, j+7]))$ obeys

$$
d_{\pi^{*} \Gamma} h_{\jmath, j+4}=h_{\jmath, j+4}\left(h_{\jmath+4} A-\pi^{*} \Gamma\right)-\left(h_{\jmath} A-\pi^{*} \Gamma\right) h_{\jmath, j+4} .
$$

By bootstrapping, we see that $h_{\jmath, \jmath+4}$ has the same estimate as (4.3). If we choose $\varepsilon>0$ so small, the argument in Lemma 4.1 can be repeated with the data $\left\{h_{j, j+4}\right\}$ to produce $h_{j}^{\prime} \in C^{\infty}(\operatorname{Aut}(Q \times[j, j+7]))$ such that $h_{j}^{\prime}\left(h_{j+4}^{\prime}\right)^{-1}=z_{j, j+4}$ $\in \mathscr{R}$ on $Z \times[j+5, j+6]$ and for some $\zeta^{\prime}>0$,

$$
\sup _{z \times[j, j+7]}\left\{\sum_{l=0}^{m}\left|\nabla_{\pi * \Gamma}^{(l)}\left(h_{j}^{\prime} A-\pi^{*} \Gamma\right)\right|^{2}\right\} \leqq \zeta^{\prime} \int_{Z \times[\jmath-1, j+8]}\left|F_{A}\right|^{2} .
$$

Now we change $h_{\jmath}^{\prime}$ to

$$
\tilde{h}_{j}=z_{0,4} \cdots z_{j-4, j} h_{j}^{\prime} .
$$

Then $\tilde{h}$, obeys the same bound as above and $\tilde{h}_{j}\left(\tilde{h}_{j_{+4}}\right)^{-1}=1$. We define $h \in$ $C^{\infty}($ Aut $(Q \times[1, T)))$ by $h=\tilde{h}$, on $Z \times[j+1, j+6]$. Then A satisfies the desired estimate.

We prove the decay estimate of the curvature of ASD connections over the cylinder $Z \times[0, \infty)$ by the parallel discussion in [7, 7.3]. The following three lemmas can be proved by replacing the trivial connection in the argument of $[7,2.3 .4,2.3 .5,2.3 .6,2.3 .7,2.3 .8]$ by the flat connection $\Gamma$. We prove the first only.

LEMMA 4.3. There are constants $N, \eta>0$ such that if $B$ is a connection on $Q$ in Coulomb gauge relative to $\Gamma$ (i.e. $\left.d_{\Gamma}^{*}(B-\Gamma)=0\right)$ and satisfies $\|B-\Gamma\|_{L^{4}}<\eta$, then $\|B-\Gamma\|_{L_{1}^{2}}=\|B-\Gamma\|_{L^{2}}+\left\|\nabla_{\Gamma}(B-\Gamma)\right\|_{L^{2}} \leqq N\left\|F_{B}\right\|_{L^{2}}$.

Proof. Since $H^{1}(Z$ Ad $Q)=0$, the basic elliptic estimate for the operator $d_{\Gamma}^{*}+d_{\Gamma}$ on 1 -forms gives a bound

$$
\|B-\Gamma\|_{L_{1}^{2} \leqq} \leqq c_{1}\left\|d_{\Gamma}(B-\Gamma)\right\|_{L^{2}} .
$$

Using the Sobolev multiplication theorem, we get

$$
\|B-\Gamma\|_{L_{1}^{2}} \leqq c_{1}\left\|F_{B}\right\|_{L^{2}}+c_{1} c_{2}\|B-\Gamma\|_{{ }_{L} L}\|B-\Gamma\|_{L_{1}^{2}} .
$$


If $\|B-\Gamma\|_{L^{4}}<1 /\left(2 c_{1} c_{2}\right)$, then we can rearrange it as

$$
\|B-\Gamma\|_{L_{1}^{2}}\left\{1-c_{1} c_{2}\|B-\Gamma\|_{L^{4}}\right\} \leqq c_{1}\left\|F_{B}\right\|_{L},
$$

to get $\|B-\Gamma\|_{L_{1}^{2}} \leqq 2 c_{1}\left\|F_{B}\right\|_{L^{2}}$.

For a connection $B$ on $Q$ and $l \geqq 1$, put:

$$
Q_{l}(B)=\left\|F_{B}\right\|_{L^{\infty}}+\sum_{j=0}^{l}\left\|\nabla_{B}^{(i)} F_{B}\right\|_{L^{2}} .
$$

LEMMA 4.4. There is a constant $\eta^{\prime}>0$ such that if the connection $B$ of Lemma 4.3 has $\|B-\Gamma\|_{L 4}<\eta^{\prime}$, then for each $l \geqq 1$, a bound,

$$
\|B-\Gamma\|_{L_{l+1}^{2}}=\sum_{k=0}^{l}\left\|\nabla_{\Gamma}^{(k)}(B-\Gamma)\right\|_{L^{2}} \leqq f_{l}\left(Q_{l}(B)\right)
$$

holds for a universal continuous function $f_{l}$, independent of $B$, with $f_{l}(0)=0$.

In the lemma below, by a one-parameter family we mean that they are smooth in the $Z$ variable, and all partial derivatives are continuous in both variables.

PROPOSITION 4.5. There is a constant $\varepsilon>0$ such that if $B_{t}^{\prime}(0 \leqq t \leqq 1)$ is a one-parameter family of connections on $Q$ with $\left\|F_{B_{t}^{\prime}}\right\|_{L^{2}}<\varepsilon$ for all $t$ and with $B_{0}^{\prime}=\Gamma$, then for each $t$ there exists a one-parameter family of gauge transformations $u_{t}$ such that $u_{0}=1$ and $u_{t}\left(B_{t}^{\prime}\right)=B_{t}$ satisfies

$$
\begin{aligned}
& d_{\Gamma}^{*}\left(B_{t}-\Gamma\right)=0, \\
& \left\|B_{t}-\Gamma\right\|_{L_{1}^{2}}<2 N\left\|F_{B_{t}}\right\|_{L^{2}},
\end{aligned}
$$

where $N$ is the constant in Lemma 4.3.

PROPOSITION 4.6. There are constants $\varepsilon>0$ and $\xi>0$ independent of $T$ such that if $A$ is an $\Lambda S D$ connection on $Q \times(-T, T)$ with $\int_{J \times(-T, T)}\left|F_{A}\right|^{2} \leqq \varepsilon$, then for all $(x, t) \in Z \times[-T+8, T-8]$,

$$
\left|F_{A}\right|_{(x, t) \leqq \zeta e^{-\lambda(T-|t|)}}\left(\int_{Z \times(-T, T)}\left|F_{A}\right|^{2}\right)^{1 / 2}
$$

Proof. We apply Proposition 4.2 over $Z \times(-T, T)$ to obtain a gauge transformation $h \in C^{\infty}($ Aut $(Q \times(-T+1, T-1)))$ such that for $-T+8 \leqq t \leqq T-8$,

$$
\sup _{Z \times[t-1, t+1]}\left\{\left.\sum_{l=0}^{1}\left|\nabla\left(\left.\pi^{*} \Gamma\left(h A-\pi^{*} \Gamma\right)\right|^{2}\right\} \leqq \zeta \int_{Z \times[t-8, t+8]}\right| F_{A}\right|^{2} .\right.
$$

We henceforth omit $h$ for simplicity. We write $A_{t}$ for the restriction of $A$ to 
$Z \times\{t\}(-T+8 \leqq t \leqq T-8)$. We take the path

$$
A_{t, s}=\Gamma+s\left(A_{t}-\Gamma\right) \quad(0 \leqq s \leqq 1)
$$

from $\Gamma$ to $A_{t}$. By (4.4) we can assume that $\left\|F_{A_{t}, s}\right\|_{L^{2}}<\varepsilon$ for small $\xi>0$. Then we apply Proposition 4.5 to get a gauge transformation $l_{t}$ on $Q$ which is homotopic to the identity and satisfies

$$
\left\|l_{t} A_{t}-\Gamma\right\|_{L_{1}^{2}}<2 N\left\|F_{A_{t}}\right\|_{L^{2}} .
$$

We use the 'Chern-Simons invariant relative to $\Gamma$ ' defined by

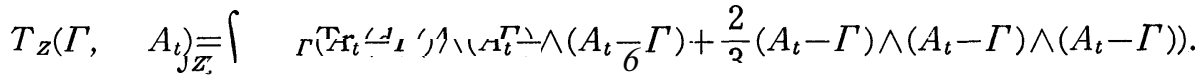

A direct calculation shows that

$$
T_{Z}\left(\Gamma, l_{t} A_{t}\right)=T_{Z}\left(\Gamma, A_{t}\right)+\frac{1}{3} \operatorname{deg} l_{t} .
$$

Here the last term

$$
\operatorname{deg} l_{t}=\int_{J Z} \operatorname{Tr}\left(d_{\Gamma} l_{t} l_{t}^{-1} \wedge d_{\Gamma} l_{t} l_{t}^{-1} \wedge d_{\Gamma} l_{t} l_{t}^{-1}\right)
$$

is a homotopy invariant by Stoke's theorem. So we have $\operatorname{deg} l_{t}=0$. For $-T+8 \leqq t \leqq T-8$, we write

$$
\nu(t)=\int_{Z \times(-t, t)}\left|F_{A}\right|^{2} .
$$

Then we can easily verify that

$$
\frac{d \nu}{d t}=2\left(\left\|F_{A_{t}}\right\|_{L^{2}}^{2}+\left\|F_{A_{-t}}\right\|_{L^{2}}^{2}\right)
$$

and

$$
\nu(t)=T_{Z}\left(A_{t}, \Gamma\right)-T_{Z}\left(A_{-t}, \Gamma\right)
$$

We need a simple lemma; any $a \in \Omega_{Z}^{1}(\operatorname{Ad} Q)$ satisfies

$$
\int_{Z} \operatorname{Tr}\left(d_{\Gamma} a \wedge a\right) \leqq \frac{1}{\lambda_{\lambda}} \int_{J \Delta}\left|d_{\Gamma} a\right|^{2} .
$$

We prove it quickly. If $a$ is replaced by $a+d_{\Gamma} f$ for some $f \in \Omega^{\circ}(\operatorname{Ad} Q)$, each of the integrals is unchanged, so we may assume that $a$ satisfies $d_{\Gamma}^{*} a=0$. Then

$$
\int_{Z} \operatorname{Tr}\left(d_{\Gamma} a \wedge a\right) \leqq\|a\|_{L^{2}}\left\|d_{\Gamma} a\right\|_{L^{2}}
$$

and $\left.\int_{z} d_{\Gamma} a\right|^{2}=\left\langle a, \Delta_{\Gamma} a\right\rangle$. So $\left\|d_{\Gamma} a\right\|_{L^{2}} \geqq \lambda\|a\|_{L^{2}}$. Since $H^{1}(Z ; \operatorname{Ad} Q)=0$, we see 
that $\int_{J z} \operatorname{Tr}\left(d_{\Gamma} a \wedge a\right) \leqq \lambda^{-1} \int_{z}\left|d_{\Gamma} a\right|^{2}$.

Using (4.5), (4.6), (4.7) and the Sobolev embedding theorem $L_{1}^{2} \rightarrow L^{4}$, we get

$$
\begin{aligned}
\left|T_{Z}\left(\Gamma, A_{t}\right)\right| & \leqq \frac{1}{\lambda}\left\|F_{A_{t}}\right\|_{L^{2}}^{2}+c\left\|l_{t} A_{t}-\Gamma\right\|_{L_{1}^{2}}^{3} \\
& \leqq \frac{1}{\lambda}\left\|F_{A_{t}}\right\|_{L^{2}}^{2}+8 c N^{3}\left\|F_{A_{t}}\right\|_{L^{2}}^{3},
\end{aligned}
$$

where the constant $c$ depends only on $Q$. So we have

$$
\frac{d \nu}{d t} \geqq 2 \lambda \nu-c\left(\frac{d \nu}{d t}\right)^{3 / 2} \text {. }
$$

We also know that $\nu$ and $d \nu / d t$ are small by (4.4). We use an elementary inequality; if $y+C y^{3 / 2} \geqq 2 \lambda x$ for some $\mathrm{C}$, and $x$ and $y$ are small, then $y \geqq 2 \lambda x$ $-C^{\prime} x^{3 / 2}$ for another constant $C^{\prime}$ '. Hence (4.8) gives

$$
\frac{d \nu}{d t} \geqq 2 \lambda \nu-c \nu^{3 / 2} \text {. }
$$

We choose $\varepsilon>0$ so small that $\delta=\varepsilon^{1 / 2} \leqq(1 / 2) \lambda$. Then the inequality above gives $d \nu / d t \geqq(2 \lambda-\delta) \nu$, which we can integrate to get an exponential bound

$$
\nu(t) \leqq e^{(2 \lambda-\delta)(t-T)} \nu(T) .
$$

Feeding back this into the differential inequality, we get

$$
\begin{aligned}
\frac{d \nu}{d t^{+}+} & 2 \lambda \nu-c \delta e^{(2 \lambda-\delta)(t-T) / 2} \nu \\
& \geqq 2 \lambda \nu-\frac{c \delta e^{(2 \lambda-\delta)(t-T) / 2}}{2 \lambda-\delta} \frac{d \nu}{d t} .
\end{aligned}
$$

It follows that

$$
\begin{aligned}
\log \nu(T)-\log \nu(t) & \geqq 2 \lambda \int_{t}^{T} \frac{d \tau}{1+c e^{(2 \lambda-\delta)(t-T) / 2}} \\
& \geqq 2 \lambda \int_{t}^{T}\left(1-c e^{(2 \lambda-\delta)(t-T) / 2}\right) d \tau \\
& \geqq 2 \lambda(T-t)-\frac{4 c \lambda}{2 \lambda-\delta} .
\end{aligned}
$$

Taking exponentials, we have the bound

$$
\nu(t) \leqq K \nu(T) e^{2 \lambda(t-T)},
$$

with $K=\exp (4 c \lambda /(2 \lambda-\delta))$. Finally we use the following for any element / in $\Omega^{2}(\operatorname{Ad}(Q \times(-1,1)))$ with $d_{A}^{*} f=d_{A} f=0$, we have an elliptic estimate: 


$$
\sup _{Z \times(0)}|f|^{2} \leqq c \int_{Z \times(-1,1)}|f|^{2} .
$$

Since $A$ obeys the uniform $C^{1}$-estimate by (4.4), we can take $c$ which is independent of $A$. Applying this to $f=F_{A}$ on $Z \times(-T+8, T-8)$, we get

$$
\left|F_{\mathrm{A}}\right|_{(x, t)} \leqq c \nu(t)^{1 / 2} \leqq c e^{\lambda\left(\mid t_{1}-T\right)} \nu(T)^{1 / 2}
$$

and the proof is completed.

We apply the above proposition over $Z \times\left(n_{0}, 2 t-n_{0}\right)$ to obtain that

COROLLARY 4.7. // $A$ is an ASD connection on $\pi^{*} Q$ with $\int_{J_{Z \times\left[n_{0}, \infty\right)}}\left|F_{A}\right|^{2}<\varepsilon$ for some $n_{0} \in N$, then for all $(x, t) \in Z \times\left[n_{0}+1, \infty\right)$,

$$
\mid F A(x, t) \leqq \zeta e^{-\lambda t}\left(\int_{Z \times\left[n_{0}, \infty\right)}\left|F_{A}\right|^{2}\right)^{1 / 2} .
$$

\section{Gluing ASD connections.}

According to [7, 7.1], we shall construct a gluing map from $\mathscr{M}_{Y_{1}}\left(l_{Y_{1}}, \sigma_{1}^{*}(\eta), g_{1}\right)$ $\times \mathscr{M}_{Y_{2}}\left(l_{Y_{2}}, \sigma_{2}^{*}(\eta), g_{2}\right)$ to $\mathscr{M}_{X_{n}}\left(l_{X_{n}}, \eta, g_{n}\right)$. To obtain the gluing map globally, we need a technical lemma below. In this section, we use $c$ for a constant independent of $n$ and use $c_{\imath}$ for a constant with respect to $Y_{\imath}$.

LEMMA 5.1. There are constants $\varepsilon>0, t_{0}=t_{0, m}>0$ and $\rho=\rho_{m}>0$ with the following significance: Let $A$ be an ASD connection on $\pi^{*} Q$. Suppose that $n_{0} \in N$ exists such that $\int_{Z \times\left[n_{1}, \infty\right)}\left|F_{A}\right|^{2}<\varepsilon$. Then there exists $h \in C^{\infty}\left(\operatorname{Aut}\left(Q \times\left[n_{0}+\right.\right.\right.$ $1, \infty))$ ) such that for $t \geqq n_{0}+t_{0}$,

$$
\begin{gathered}
\sup _{z \times[t-1, t+1]}\left\{\sum_{l=0}^{m}\left|\nabla_{\pi * \Gamma}^{(l)}\left(h A-\pi^{*} \Gamma\right)\right|^{2}\right\} \leqq \rho e^{-2 \lambda\left(t-n_{0}\right)}, \\
i_{\partial / \partial t}\left(h A-\pi^{*} \Gamma\right)=0 .
\end{gathered}
$$

Such $a h$ is unique up to $\mathbb{R}$. Moreover, if $A$ satisfies

$$
\left.J_{J \times(0,0)} \sum_{l=0} \nabla_{\pi * \Gamma}^{(l)}\left(A-\pi^{*} \Gamma\right)\right|^{2}<\infty,
$$

then we can choose $h$ so that $\left.h\right|_{t=n_{0}}$ is homotopic to the identity, and such $a h$ is unique.

Proof. By Proposition 4.2 and Corollary 4.7, there exists $h \in C^{\infty}($ Aut $(Q \times$ $\left.\left.\left[n_{0}+1, \infty\right)\right)\right)$ such that for $t \geqq n_{0}+8$, 


$$
\sup _{\boldsymbol{z} \times[t-1, t+1]}\left\{\sum_{l=0}^{m}\left|\nabla_{\pi * \Gamma}^{(l)}\left(h A-\pi^{*} \Gamma\right)\right|^{2}\right\} \leqq c e^{-2 \lambda\left(t-n_{0}\right)} .
$$

Solve the ordinary differential equation for $\tilde{h} \in C^{\infty}\left(\right.$ Aut $\left.\left(Q \times\left[n_{0}+1, \infty\right)\right)\right)$ :

$$
i_{\partial / \partial t}\left(\hat{h}(h A)-\pi^{*} \Gamma\right)=-\frac{\partial}{\partial t} \tilde{h} \tilde{h}^{-1}+\tilde{h} i_{\partial / \partial t}\left(h A-\pi^{*} \Gamma\right) \tilde{h}^{-1}=0
$$

with initial condition $\lim _{t \rightarrow \infty} \tilde{h}=1$. From the differential equation we have

$$
-\frac{\partial}{\partial t}\left(d_{\Gamma} \tilde{h}\right)=d_{\Gamma} \tilde{h} i_{\partial / \partial t}\left(h A-\pi^{*} \Gamma\right)+\tilde{h} d_{\Gamma}\left(i_{\partial / \partial t}\left(h A-\pi^{*} \Gamma\right)\right) \cdot
$$

This gives us

$$
-\frac{\partial}{\partial t}\left|d_{\Gamma} \tilde{h}\right|^{2} \leqq c e^{-\lambda\left(t-n_{0}\right)}\left(\left|d_{\Gamma} \tilde{h}\right|^{2}+\left|d_{\Gamma} \tilde{h}\right|\right),
$$

which integrates over $[t-1, \infty)$ to get an inequality

$$
\left\|d_{\Gamma} \tilde{h}\right\|_{C^{0}(Z \times[t-1, \infty))}^{2} \leqq c e^{-\lambda\left(t-n_{0}\right)}\left(\left\|d_{\Gamma} \tilde{h}\right\|_{C 0(Z \times[t-1, \infty))}^{2}+\left\|d_{\Gamma} \tilde{h}\right\|_{C 0(Z \times[t-1, \infty))}\right) .
$$

For $t>n_{0}+t_{0}$ with $c e^{-2 \lambda t_{0}}<1 / 2$, we can rearrange this to get

$$
\left\|d_{\Gamma} \tilde{h}\right\|_{C 0(Z \times[t-1, t+1)} \leqq\left\|d_{\Gamma} \tilde{h}\right\|_{C 0(Z \times[t-1, \infty))} \leqq 2 c e^{-\lambda\left(t-n_{0}\right)} .
$$

Now we can bootstrap the equation

$$
\tilde{h}(h A)-\pi^{*} \Gamma=-d_{\Gamma} \tilde{h} \tilde{h}^{-1}-\tilde{h} \imath_{\partial / \partial t}\left(h A-\pi^{*} \Gamma\right) \tilde{h}^{-1}+\tilde{h}\left(h A-\pi^{*} \Gamma\right) \tilde{h}^{-1} .
$$

Then we see that $h^{\prime}=\tilde{h} h$ satisfies the desired properties.

If $h^{\prime}$ also satisfies the properties of Lemma 5.1, then $h^{\prime} h^{-1}$ is independent of $t$ and satisfies

$$
d_{\Gamma}\left(h^{\prime} h^{-1}\right)=h^{\prime} h^{-1} i_{\partial / \partial t}\left(h A-\pi^{*} \Gamma\right)-i_{\partial / \partial t}\left(h^{\prime} A-\pi^{*} \Gamma\right) h^{\prime} h^{-1} .
$$

This implies that $h^{\prime} h^{-1}$ converges to a flat gauge as $t \rightarrow \infty$. So $h^{\prime} h^{-1}$ must be an element in $\mathcal{R}$.

If $A$ satisfies the additional condition, then by the equation

$$
d_{\pi^{*} \Gamma} h=h\left(A-\pi^{*} \Gamma\right)-\left(h A-\pi^{*} \Gamma\right) h
$$

and the Sobolev embedding theorem $L_{3}^{2} \rightarrow C^{0}$, we get

$$
\left\|d_{\pi^{*} \Gamma} h\right\|_{C^{0}([t, t+1])}->0(t \rightarrow \infty) .
$$

Hence A factors as $h=r(h) \exp \xi$ with $r(h) \in \mathscr{R}$ and $\xi \in C^{\infty}\left(\operatorname{Ad}\left(Q \times\left[n_{1}, \infty\right)\right)\right)$ for some $n_{1} \in \boldsymbol{N}$. So $h^{\prime}=r(h)^{-1} h$ has the desired properties and it is unique, since the primary difference $\mathrm{b}(1, \mathrm{r})$ is non-zero for any non-identity element $r \in \mathcal{R}$.

The above unique gauge is said to be exponential gauge. We combine Lemma 5.1 with Lemma 3.1 to deduce 
COROLLARY 5.2. $/ / B_{2}$ is an ASD connection in $\mathcal{A}_{P_{2}}$ then there are $n_{0} \in \boldsymbol{N}$ and $u_{i} \in \mathcal{G}_{P_{\imath}}$ such that $A_{i}=u_{i} B_{\imath}$ satisfies the following conditions.

(i) $A$ is smooth over $\tau_{\imath}^{-1}\left(\left[n_{0}+1, \infty\right)\right)$

(ii) For $t \geqq n_{0}+t_{0}$,

$$
\begin{gathered}
\sup _{Z \times[t-1, \mathrm{i}+1]}\left(4_{l=0}^{m} \sum\left|\nabla_{\pi * \Gamma}^{(l)}\left(A_{i}-\pi^{*^{\prime}}\right)\right|^{2}\right\} \leqq \rho e^{-2 \lambda\left(t-n_{0}\right)}, \\
i_{\partial \partial \partial t}\left(A_{i}-\pi^{*} \Gamma\right)=0 .
\end{gathered}
$$

$/ / A_{\imath}^{\prime}$ also satisfies the above condition. then $A_{\imath}=A_{\imath}^{\prime}$ over $\tau_{\imath}^{-1}\left(\left[n_{0}+t_{0}, \infty\right)\right)$

Let $\beta_{i}, \gamma_{\imath}$ and $\mu_{\imath}$ be smooth cut off functions satisfying

$$
\begin{aligned}
\beta_{i}(x, t) & = \begin{cases}1 & \text { on } \tau_{\imath}^{-1}([0, n+1]), \\
(n+N+1-t) / N & \text { on } \tau_{\imath}^{-1}([n+1+\varepsilon, n+N+1-\varepsilon]),\end{cases} \\
\gamma_{\imath} & = \begin{cases}1 & \text { on } \tau_{\imath}^{-1}([0, n-1]), \\
0 & \text { on } \tau_{\imath}^{-1}([n+1, \infty)),\end{cases} \\
\mu_{\imath} & = \begin{cases}1 & \text { on } \tau_{\imath}^{-1}([0, n-N-2]), \\
0 & \text { on } \tau_{\imath}^{-1}([n-N-1, \infty)),\end{cases} \\
\gamma_{1}(x, t)+\gamma_{2}(n-x, t)=1 & \text { if }(x, t) \in \tau_{\imath}^{-1}([n-1, n+1]),
\end{aligned}
$$

for a small $0<\varepsilon<1$. Then a direct calculation shows that

$$
\left\|\nabla \beta_{i}\right\|_{L^{q}\left(Y_{i}\right)} \leqq K N^{-(q-1) / q},
$$

where $K$ is independent of a and $N$. For $A_{i} \in \mathcal{A}_{P_{i}}$, we define a connection $A_{2}^{\prime}$ on $P_{\imath}$ by $A_{i}^{\prime}=\mu_{i}\left(A_{i}-\pi^{*} \Gamma\right)+\left(1-\mu_{i}\right) \pi^{*} \Gamma$ and a connection $A^{\prime}$ on $P_{n}$ by

$$
J_{n}\left(A_{1}, A_{2}\right)=A^{\prime}= \begin{cases}A_{1}^{\prime} & \text { over } \tau_{1}^{-1}([0, n+1]), \\ A_{2}^{\prime} & \text { over } \tau_{2}^{-1}([0, n+1]) .\end{cases}
$$

LEMMA 5.3. // $A_{2}$ is an ASD connection in $\mathcal{A}_{P_{2}}$ satisfying the conditions (i) (ii), then there is a constant $c>0$ such that

(1) $\left\|A_{i}-A_{i}^{\prime}\right\|_{L^{q}{ }_{\left(Y_{i}\right)}<c e^{-\lambda(n-N)}}$

(2) $\left\|A_{i}-A_{i}^{\prime}\right\|_{L_{\tilde{\delta}}^{p}\left(Y_{i}\right)}+\left\|\nabla_{\pi^{*} \Gamma}\left(A_{i}-A_{i}^{\prime}\right)\right\|_{L_{\tilde{\delta}}^{p}\left(Y_{i}\right)}<c e^{-(\lambda-\delta / p)(n-N)}$,

(3) $\left\|F_{A_{i}^{\prime}}^{+}\right\|_{L_{\delta}^{p}\left(Y_{i}\right)}<c e^{-(\lambda-\delta / p)(n-N)}$.

Proof. This is obvious.

We are in the position to argue the right inverse to $d_{A^{\prime}}^{+}$. For an ASD connection $A_{\imath}$ in $\mathcal{A}_{P_{i}}$, we take the Laplacian 


$$
\Delta_{A_{\imath}}=d_{A_{i}}^{+} e^{-\tau_{i} \delta}\left(d_{A_{i}}^{+}\right) * e^{\tau_{i} \delta}: L_{2, \delta}^{p}\left(Y_{\imath}\right) \longrightarrow L_{\delta}^{p}\left(Y_{\imath}\right) .
$$

The condition $H_{A_{\imath}}^{2}=0$ implies that $\Delta_{A_{\imath}}$ is invertible [15, Lemma 7.3]. Then there is the right inverse $P_{\imath}$ to the operator $d_{A}^{+}$

$$
P_{\imath}=e^{-\tau_{i} \delta}\left(d_{A_{i}}^{+}\right){ }^{*} e^{\tau_{i} \delta}\left(\Delta_{A_{i}}\right)^{-1}: L_{\delta}^{p}\left(Y_{\imath}\right) \longrightarrow L_{1, \delta}^{p}\left(Y_{\imath}\right),
$$

which satisfies $\left\|P_{i} \xi\right\|_{L^{p}\left(Y_{i}\right)} \leqq c_{i}\|\xi\|_{L_{j}^{p}\left(Y_{i}\right)}$ for some constant $c_{\imath}$. Composing with the Sobolev embedding

$$
L_{1, \delta}^{p}\left(Y_{\imath}\right) \longrightarrow L_{q \delta / p}^{q}\left(Y_{i}\right)
$$

[15, Lemma 7.2], we have $\left\|P_{i} \xi\right\|_{L_{\boldsymbol{y}^{\prime}}{ }^{q}\left(Y_{i}\right)} \leqq c_{i}\|\xi\|_{\mathcal{S}_{\tilde{j}}^{q}\left(Y_{i}\right)}$ We also need Hölder's inequality, for 1 -forms $a$, ft,

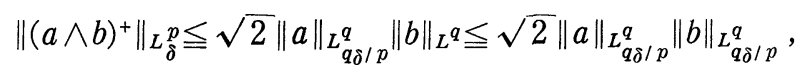

over $X_{n}$ or $Y_{\imath}$. Now let $Q_{\imath}: L_{\delta}^{p}\left(X_{n}\right) \rightarrow L_{1, \delta}^{p}\left(X_{n}\right)$ e the operator defined by

$$
Q_{i} \xi=\beta_{i} P_{i} \gamma_{i}(\xi) \text {. }
$$

Then we obtain a bound $\left\|Q_{i} \xi\right\|_{L_{1, \delta}^{p}}^{p}\left(x_{n}\right) \leqq c_{i} e^{2 \delta}\|\xi\|_{L_{\delta}^{p}\left(X_{n}\right)}$.

LEMMA 5.4. There is a constant $\varepsilon_{i}=\varepsilon_{i}(N, n) \rightarrow 0$ as $n \rightarrow \infty$ and $N \rightarrow \infty$ in order such that

$$
\left\|\gamma_{i} \xi-d_{A_{i}^{\prime}}^{+} Q_{i} \xi\right\|_{L_{\delta}^{p}\left(X_{n}\right)} \leqq \varepsilon_{i}(N, n)\|\xi\|_{L_{\delta}^{p}\left(X_{n}\right)}
$$

Proof. If we write $A_{\imath}^{\prime}=A_{i}+a_{i}$, then

$$
\begin{aligned}
d_{A_{i}^{\prime}}^{+}\left(Q_{i} \xi\right) & =d_{A_{i}+a_{i}}^{+}\left(\beta_{i} P_{i}\left(\gamma_{i} \xi\right)\right) \\
& =\beta_{i}\left(d_{A_{i}}^{+} P_{i}\left(\gamma_{i} \xi\right)\right)+\left(\nabla \beta_{i}\right) P_{i}\left(\gamma_{i} \xi\right)+\left[\beta_{i} a_{\imath}, P_{i}\left(\gamma_{i} \xi\right)\right] \\
& =\gamma_{i} \xi+\left(\nabla \beta_{i}\right) P_{i}\left(\gamma_{i} \xi\right)+\left[\beta_{i} a_{\imath}, P_{i}\left(\gamma_{i} \xi\right)\right] .
\end{aligned}
$$

By Lemma 5.3, we get

$$
\begin{aligned}
& \left\|\left(\nabla \beta_{i}\right) P_{i}\left(\gamma_{i} \xi\right)\right\|_{L_{\delta}^{p}\left(X_{n}\right)} \leqq\left\|\left(\nabla \beta_{i}\right) P_{i}\left(\gamma_{i} \xi\right)\right\|_{L_{\delta}^{p}\left(Y_{i}\right)} \\
& \leqq \sqrt{2}\left\|\nabla \beta_{i}\right\|_{L^{q}\left(Y_{i}\right)}\left\|P_{i}\left(\gamma_{i} \xi\right)\right\|_{L_{q_{\delta} / p}^{q}}\left(Y_{i}\right) \\
& \leqq c_{i} K N^{-(q-1) / q} e^{2 \delta}\|\xi\|_{L_{\delta}^{p}\left(X_{n}\right)}, \\
& \left\|\left[\beta_{i} a_{\imath}, P_{i}\left(\gamma_{i} \xi\right)\right]\right\|_{L_{\delta}^{p}\left(x_{n}\right) \leqq\left\|\left[\beta_{i} a_{\imath}, P_{i}\left(\gamma_{i} \xi\right)\right]\right\|_{\delta}^{p}\left(Y_{i}\right)} \\
& \leqq \sqrt{2}\left\|\beta_{i} a_{i}\right\|_{L^{q}\left(Y_{i}\right)}\left\|P_{i}\left(\gamma_{i} \xi\right)\right\|_{L_{q_{\delta} / p}^{q}\left(Y_{i}\right)} \\
& \leqq c_{i} e^{-\lambda(n-N)+2 \delta}\|\xi\|_{L_{\delta}^{p}\left(X_{n}\right)} .
\end{aligned}
$$

The result now follows by letting $\varepsilon_{\imath}=c_{i} e^{2 \delta}\left(e^{-\lambda(n-N)}+K N^{-(q-1) / q}\right)$. 
We put

$$
Q=Q_{1}+Q_{2}: L_{\delta}^{p}\left(X_{n}\right) \longrightarrow L_{1, \delta}^{p}\left(X_{n}\right) .
$$

The operator $R=d_{A}^{+} Q-1$ obeys a bound

$$
\|R(\xi)\|_{L_{\delta}^{p}\left(X_{n}\right)} \leqq\left(\varepsilon_{1}(N, n)+\varepsilon_{2}(N, n)\right)\|\xi\|_{L_{\delta}^{p}\left(X_{n}\right)} .
$$

We choose $N_{0}$ and $n_{0}=n_{0}\left(N_{0}\right)$ so that $\varepsilon_{i}\left(N_{0}, n\right) \leqq 1 / 3$ for all $n \geqq n_{0}$. Then the operator norm of $R$ is at most $2 / 3$. So $1+R$ is invertible and the norm of the inverse is at most 3. Thus the right inverse $P=Q(1+R)^{-1}$ to $d_{A}^{+}$satisfies

$$
\begin{aligned}
&\|P \xi\|_{L_{1, \delta}^{p}\left(X_{n}\right)} \leqq\left\|Q_{1}(1+R)^{-1} \xi\right\|_{L_{1 j}^{p}\left(X_{n}\right)}+\left\|Q_{2}(1+R)^{-1} \xi\right\|_{L_{1, \delta}^{p}\left(X_{n}\right)} \\
& \\
& \leqq c_{1} e^{2 \delta}\left\|(1+R)^{-1} \xi\right\|_{L_{\delta}^{p}\left(X_{n}\right)}+c_{2} e^{2 \delta}\left\|(1+R)^{-1} \xi\right\|_{L_{\delta}^{p}\left(x_{n}\right)} \\
& \leqq c\|\xi\|_{L_{\delta}^{p}\left(x_{n}\right)}
\end{aligned}
$$

with $c=3 e^{2 \delta}\left(c_{1}+c_{2}\right)$. Combining with the Sobolev embedding, we get $\left.\|P \xi\|_{L_{\alpha_{\delta} / p}^{q}} a x_{n}\right) \leqq c\|\xi\|_{L_{s}^{p}\left(X_{n}\right)}$. We seek a solution $A^{\prime}+a$ to the ASD equations in the form $a=P(\xi)$. If we write $q(\xi)=(P \wedge P \xi)^{+}$, the ASD equation becomes

By Hölder's inequality,

$$
\xi+q(\xi)=-F_{A}^{+}
$$

$$
\left\|q\left(\boldsymbol{\xi}_{1}\right)-q\left(\boldsymbol{\xi}_{2}\right)\right\|_{L_{\delta}^{p}\left(x_{n}\right)} \leqq \sqrt{2} c^{2}\left\|\boldsymbol{\xi}_{1}-\boldsymbol{\xi}_{2}\right\|_{L_{\delta}^{p}\left(x_{n}\right)}\left\{\left\|\boldsymbol{\xi}_{1}\right\|_{L_{\delta}^{p}\left(x_{n}\right)}+\left\|\boldsymbol{\xi}_{2}\right\|_{L_{\delta}^{p}\left(x_{n}\right)}\right\} .
$$

LEMMA 5.5. ([7, Lemma (7.2.23)] Let $5: B \rightarrow B$ be a smooth map on a Banach space and $\left\|S \xi_{1}-S \xi_{2}\right\| \leqq k\left\{\left\|\xi_{1}\right\|+\left\|\xi_{2}\right\|\right\}\left\|\xi_{1}-\xi_{2}\right\|$ for some $k>0$ and all $\boldsymbol{\xi}_{1}, \boldsymbol{\xi}_{2} \in B$. Then for each $\eta \in B$ with $\|\eta\|<1 /(10 k)$ there is a unique $\xi$ with $\|\boldsymbol{\xi}\| \leqq 1 /(5 k)$ such that

$$
\xi+S(\xi)=\eta \text {. }
$$

We apply Lemma 5.5 to the above equation with $S=q, \eta=-F_{A^{\prime}}^{+}$and $k \geqq$ $\sqrt{2} c^{2}$. Then

PROPOSITION 5.6. Let $A_{\imath}$ be an ASD connection in $\mathcal{A}_{P_{\imath}}$ satisfying the conditions ( $\mathrm{i}$ ), (ii). Then there exists $N_{0}$ and $n_{0}=n_{0}\left(N_{0}\right)$ such that for all $n \geqq n_{0}$, we can find an ASD connection $I_{n}\left(A_{1}, A_{2}\right)=A^{\prime}+a$ on $P_{n}$ with $\|a\|_{L}^{q} q \delta / p\left(x_{n}\right) \leqq$ $c\|a\|_{L_{1}^{p}, \delta\left(X_{n}\right)} \leqq c e^{-(\lambda-\delta / p)\left(n-N_{0}\right)}$. a is the unique such solution which can be written in the form $P \xi$. If $u_{\imath} A_{\imath}$ also satisfies the conditions ( $\mathrm{i}$ ), (ii) for some $u_{i} \in \mathcal{G}_{P_{i}}$, then

$$
I_{n}\left(u_{1} A_{1}, u_{2} A_{2}\right)=\left(u_{1} \cup u_{2}\right) I_{n}\left(A_{1}, A_{2}\right) .
$$

Moreover we can choose $N_{0}$ and $n_{0}=n_{0}\left(N_{0}\right)$ so that for all $n \geqq n_{0}, A_{t}=A^{\prime}+t a$, $(0 \leqq t \leqq 1)$ is regular, that is $\left[A^{\prime}\right] \in U\left(I_{n}\left(A A_{2}\right)\right)$.

Proof. By bootstrapping (5.4), we see that the solutions we solved are in 
$C^{\infty}$ if so are $A_{2}$. The second part is obvious from the gauge equivalence of the above construction. We prove the last part. By (5.3), we have

$$
\left\|\left(d_{A_{t}}^{+}-d_{A^{\prime}}^{+}\right) \xi\right\|_{L_{\delta}^{p}\left(X_{n}\right)}=\|t[a, \xi]\|_{L_{\delta}^{p}\left(X_{n}\right)} \leqq c e^{-(\lambda-\delta / p)\left(n-N_{0}\right)}\|\xi\|_{L_{1, \delta}^{p}\left(X_{n}\right)} .
$$

If we choose $n_{0}$ so large that $c e^{-(\lambda-\delta / p)\left(n_{0}-N_{0}\right)} \leqq 1 / 2$ then for $n \geqq n_{0}$, the operator norm of $\left(d_{\boldsymbol{A}_{t}}^{+}-d_{\boldsymbol{A}^{\prime}}^{+}\right) P$ is at most $1 / 2$ and $d_{\boldsymbol{A}_{t}}^{+} P=1+\left(d_{\boldsymbol{A}_{t}}^{+}-d_{\boldsymbol{A}^{\prime}}^{+}\right) P$ is invertible. So $A^{\prime} t$ is regular.

Now we obtain the gluing map

$$
I_{n}: \mathscr{M}_{Y_{1}}\left(l_{Y_{1}}, \rho_{1}^{*}(\eta), g_{1}\right) \times \mathscr{M}_{Y_{2}}\left(l_{Y_{2}}, \sigma_{2}^{*}(\eta), g_{2}\right) \longrightarrow \mathscr{M}_{X_{n}}\left(l_{X_{n}}, \eta, g_{n}\right),
$$

for large $N \geqq N_{0}$ and $n \geqq n_{0}\left(N_{0}\right)$. Here $N_{0}, n_{0}\left(N_{0}\right)$ are the maximal values of all $\left[A_{i}\right]$ in $\mathscr{M}_{Y}\left(l_{Y_{i}}, \boldsymbol{\sigma}_{i}^{*}(\eta), g_{i}\right)$. We may suppose that $N_{0}=0$.

We prove that $I_{n}$ is injective for large $n$. Let $A_{\imath}, B_{\imath}$ be as above. Suppose that $I_{n}\left(A_{1}, A_{2}\right)=A^{\prime}+a$ is gauge equivalent to $I_{n}\left(B_{1}, B_{2}\right)=B^{\prime}+b$ by some gauge $u_{n} \in \mathcal{G}_{X_{n}}$. We expand the equation $u_{n}\left(A^{\prime}+a\right)=B^{\prime}+b$ over $\tau_{\imath}^{-1}([n-3, n-2])$. Then

$$
a_{\pi^{*} \Gamma} u_{n}=u_{n}\left(A_{i}-\pi^{*} \Gamma\right)+u_{n} a-\left(B_{i}-\pi^{*} \Gamma\right) u_{n}-b u_{n} .
$$

By Lemma 5.3,

$$
\left\|d_{\pi^{*} \Gamma} u_{n}\right\|_{C 0\left(\tau_{i}^{-1}([n-3, n-2])\right)} \leqq c\left\|\left.d_{\pi^{*} \Gamma} u_{n}\right|_{\tau_{i}^{-1}([n-3, n-2])}\right\|_{L_{1}^{p}, \delta^{\left(Y_{i}\right)}} \leqq c e^{-(\lambda-\delta / p) n} .
$$

Hereafter $\|\mathrm{I}\|_{\left.L_{k, \delta^{(}}^{p}\right)}$ is the integral over the restriction. This implies that for large $\mathrm{n}, u_{n}$ factors as $u_{n}=r\left(u_{n}\right) \exp h_{\imath n}$ over $\tau_{\imath}^{-1}([n-3, n-2])$, where $r\left(u_{n}\right) \in \mathscr{R}$ satisfies

$$
\left\|r\left(u_{n}\right)^{-1} u_{n}-1\right\|_{C 0\left(\tau_{\imath}^{-1}([n-3, n-2])\right)} \leqq c\left\|d_{\pi^{*} \Gamma} u_{n}\right\|_{C 0\left(\tau_{\imath}^{-1}([n-3, n-2])\right)} \leqq c e^{-(\lambda-\delta / p) n} .
$$

Lemma 3.1 asserts that $r\left(u_{n}\right)$ is the identity. Since the exponential map on $\mathfrak{g} \mathfrak{0}(3)$ is a local diffeomorphism, we get

$$
\left\|h_{\imath n}\right\|_{C 0\left(\tau_{\imath}^{-1}([n-3, n-2])\right)}+\left\|d_{\pi^{*} \Gamma} h_{\imath n}\right\|_{C 0\left(\tau_{\imath}^{-1}([n-3, n-2])\right)} \leqq c e^{-(\lambda-\delta / p) n} .
$$

We put

$$
u_{\imath n}=\left\{\begin{array}{lll}
u_{n} & \text { over } & \tau_{\imath}^{-1}([0, n-3]), \\
\exp \left(\delta_{i} h_{\imath n}\right) & \text { over } & \tau_{\imath}^{-1}([n-3, \infty)),
\end{array}\right.
$$

where $\boldsymbol{\delta}_{i}$ is a cut off function such that $\boldsymbol{\delta}_{i}=1$ on $\tau_{i}^{-1}([0, n-3])$ and $\boldsymbol{\delta}_{i}=0$ on $\tau_{\imath}^{-1}([n-2, \infty))$. By estimating all the term in the right hand side of the equation

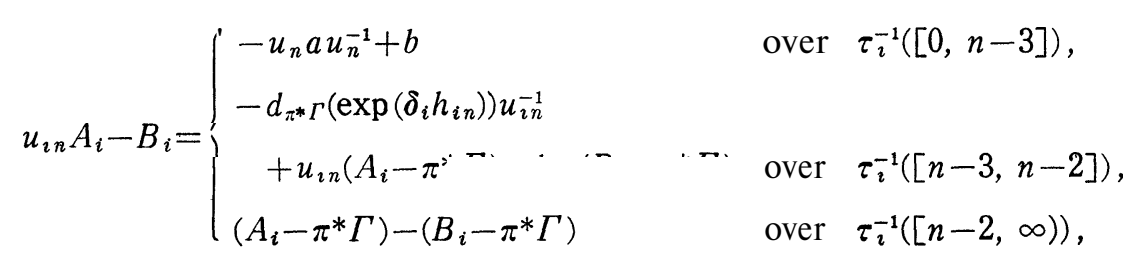


we see that $u_{i n} A_{\imath}$ converges to $B_{\imath}$ in $L_{\delta}^{p}\left(Y_{\imath}\right)$ as $n \rightarrow \infty$. On the other hand, $\mathscr{M}_{Y_{i}}\left(l_{Y_{i}}, \sigma_{i}^{*}(\eta), g_{i}\right)$ consists of isolated points with respect to the metric (c. f. [7, Lemma (4.2.4)])

This implies that $\left[A_{\imath}\right]=\left[B_{\imath}\right]$

$$
d_{P_{i}}([A],[B])=\inf _{u \in \mathcal{G}_{P_{\imath}}}\|A-u B\|_{L_{\delta}^{p}\left(Y_{i}\right)} .
$$

We will prove that $I_{n}$ is surjective for larger $n$. For a moment, we work with the space of $L_{1}^{p}$ connections $\mathcal{A}_{P_{n}}^{\prime}, L_{2}^{p}$ gauge group $\mathcal{G}_{P_{n}}^{\prime}$ and the quotient $\mathscr{B}_{P_{n}}^{\prime}=\mathcal{A}_{P_{n}}^{\prime} / \mathcal{G}_{P_{n}}^{\prime}$. Let $d_{P_{n}}$ be the metric in $\mathscr{B}_{P_{n}}^{\prime}$ given by [7, Lemma (4.2.4)]

$$
d_{P_{n}}([A],[B])=\inf _{u \in \mathcal{G}_{P}^{\prime}}\|A-u B\|_{L_{q}^{q} \delta / p}\left(X_{n}\right) .
$$

We define

$$
J_{n}: \mathscr{M}_{Y_{1}}\left(l_{Y_{1}}, \sigma_{1}^{*}(\eta), g_{1}\right) \times \mathscr{M}_{Y_{2}}\left(l_{2}, \sigma_{2}^{*}(\eta), g_{2}\right) \longrightarrow \mathscr{B}_{P_{n}}^{\prime}
$$

by $J_{n}\left(\left[A_{1}\right],\left[A_{2}\right]\right)=\left[J_{n}\left(A_{1}, A_{2}\right)\right]=\left[A^{\prime}\right]$ For $\nu>0$, let $U(\nu) \subset \mathscr{B}_{P_{n}}^{\prime}$ be the open set

$$
U(\nu)=\left\{[A] \in \mathscr{B}_{P_{n}}^{\prime} \mid d_{P_{n}}\left([A], \operatorname{Im} J_{n}\right)<\nu,\left\|F_{A}^{+}\right\|_{L_{\delta}^{p}\left(x_{n}\right)}<\nu^{3 / 2}\right\} .
$$

The solutions we have constructed lie in $U(\nu)$, if $n \geqq n_{0}=n_{0}(\nu)$ with $c e^{-(\lambda-\delta / p) n_{0}}<$ ข. Conversely.

PROPOSITION 5.7. There is a constant $\nu_{0}>0$ such that for $0<\nu<\nu_{0}$, some $n_{0}=$ $n_{0}(\nu) \in N$ satisfies the following If $n>n_{0}$, then $[A] \in U^{\prime}(\nu)$ can be represented by a connection $A$ of the form $A^{\prime}+P \xi$ with $\xi \in L_{\delta}^{p}\left(X_{n}\right)$ and $\|\xi\|_{L} p_{\delta}^{p}\left(X_{n}\right)<1 /(5 k)$ $\left(k=\sqrt{2} c^{2}\right)$.

Proof. Let $B$ be an element of $U(\boldsymbol{\nu})$. Then there is a connection $\left[A^{\prime}\right] \epsilon$ $\operatorname{Im} J_{n}$ with

$$
\left\|A^{\prime}-B\right\|_{L}^{q} \underset{q}{q} / p\left(X_{n}\right)<\nu .
$$

We write $B=A^{\prime}+b$ and consider the path

$$
B_{t}=A^{\prime}+t b \quad(0 \leqq t \leqq 1),
$$

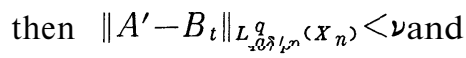

So

$$
F_{B_{t}}^{+}=(1-t) F_{A^{\prime}}^{+}+t F_{B}^{+}+\left(t^{2}-t\right)(b \wedge b)^{+} .
$$

$$
\begin{aligned}
\left\|F_{B_{t}}^{+}\right\|_{L_{q \delta / p}^{q}}^{q}\left(X_{n}\right) & \leqq(1-t)\left\|F_{A^{\prime}}^{+}\right\|_{L}^{q} \underset{q \delta / p}{q}\left(X_{n}\right)+t\left\|F_{B}^{+}\right\|_{L}^{q} \underset{q \delta / p}{q}\left(X_{n}\right)+\sqrt{2}\left(t-t^{2}\right)\|b\|_{L}^{2} \underset{q \delta / p}{q}\left(X_{n}\right) \\
& \leqq(1-t) c e^{-(\lambda-q \delta / p) n}+t \nu^{3 / 2}+\sqrt{2}\left(t-t^{2}\right) \nu^{2} .
\end{aligned}
$$

Thus we can find $\nu_{0}>0$ with the following: For $0<\nu<\nu_{0}$, there is a constant $n_{0}=n_{0}(\nu) \in N$ such that if $n>n_{\iota}$, then $\left[B_{t}\right](0 \leqq t \leqq 1)$ is contained in $U(\boldsymbol{\nu})$. We 
define $S \subset[0,1]$ to be the set of times for which there are $u_{t} \in \mathcal{G}_{P_{n}}^{\prime}$ and $\left[A^{\prime}\right] \in$ $\operatorname{Im} J_{n}$ such that

$$
u_{t} B_{t}=A^{\prime}+P \xi
$$

with $\|\xi\|_{L_{\delta}^{p}\left(x_{n}\right)}<1 /(5 k)$. We will prove that $S$ is closed and open. Suppose that $t$ is in $S$. We may take $u_{t}=1$. Then the representation $B_{t}=A^{\prime}+P \xi$ gives

$$
F_{B_{t}}^{+}=F_{A^{\prime}}^{+}+\xi+(P \xi \wedge P \xi)^{+} .
$$

This gives a bound

$$
\begin{aligned}
\|\xi\|_{L} p_{\delta}\left(X_{n}\right) & \leqq\left\|F_{B_{t}}^{+}\right\|_{L} p_{\delta}\left(X_{n}\right)+\left\|F_{A^{\prime}}^{+}\right\|_{L} p_{\delta}^{p}\left(X_{n}\right)+\sqrt{2}\|P \xi\|_{L}^{2} q \dot{q} \delta / p \\
& \leqq \nu^{3 / 2}+c e^{-(\lambda-\delta / p) n}+\sqrt{2} c^{2}\|\xi\|_{L}^{2} p_{\delta}^{\left(X_{n}\right)} .
\end{aligned}
$$

Arranging this, we get

$$
\|\xi\|_{L} p_{\delta}\left(X_{n}\right) \leqq \frac{5}{4}\left(\nu^{3 / 2}+c e^{-(\lambda-\delta / p) n}\right) .
$$

This implies that for larger $n_{0} \in \boldsymbol{N}$ and smaller $\nu_{0}>0,\|\xi\|_{L_{\delta}^{p}}\left(x_{n}\right) \leqq 1 /(10 k)$. That

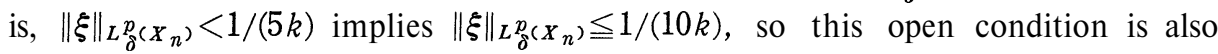
closed. We will prove that $\mathrm{S}$ is closed. Suppose that $\left\{t_{i}\right\}$ is in $\mathrm{S}$ with $t_{i} \rightarrow t$. Then we have connections $A_{t_{\imath}}=A^{\prime}+P \xi_{\imath}$ with $\left\|\xi_{i}\right\|_{L_{\delta}^{p}\left(x_{n}\right)}$, and $u_{i} \in \mathcal{G}_{P_{n}}^{\prime}$ with $u_{i} B_{t_{\imath}}=A_{t_{i}}$. By the uniform convergence bound on the $\xi_{i}$ above, we may suppose that, taking a subsequence, the $\xi_{2}$ converge to a limit $\xi$, weakly in $L_{\delta}^{p}\left(X_{n}\right)$, with $\|\xi\|_{L} p_{\delta}\left(X_{n}\right)<1 /(5 k)$ Then the connections $A_{t_{\imath}}$ converge weakly in $L_{1, \delta}^{p}\left(X_{n}\right)$ and the equation

$$
d_{B_{t}} u_{i}=u_{i}\left(B_{t_{t}}-B_{t}\right)-\left(A_{t_{\imath}}-B_{t}\right) u_{\imath}
$$

implies that, after taking a subsequence, $u_{\imath}$ converges to a limit $u$ weakly in $L_{2, \delta}^{p}\left(X_{n}\right)$. The gauge relation is preserved under weak limit, so

$$
u B_{t}=A^{\prime}+P \xi
$$

and $t$ is in 5. We will prove that $\mathrm{S}$ is open. Suppose that $t$ is in $S$. Then $B_{t}=A^{\prime}+P \xi$ with $\|\xi\|_{L_{\delta}^{p}\left(x_{n}\right)}<1 /(10 k)$. We define a map

by

$$
M: \Omega_{X_{n}}^{0}\left(\operatorname{Ad} P_{n}\right) X \Omega_{X_{n}}^{+}\left(\operatorname{Ad} P_{n}\right) \longrightarrow \Omega_{X_{n}}^{1}\left(\operatorname{Ad} P_{n}\right)
$$

$$
M(\chi, \eta)=\left(\exp (\chi)\left(A^{\prime}+P(\xi+\eta)\right)-B_{t} .\right.
$$

Let $B_{1}$ be the completion of $\Omega_{X_{n}}^{0}\left(\operatorname{Ad} P_{n}\right) \times \Omega_{X_{n}}^{+}\left(\operatorname{Ad} P_{n}\right)$ in the norm :

$$
\|(\chi, \eta)\|_{B_{1}}=\left\|d_{A^{\prime}} \chi\right\|_{L_{q \delta / p}^{q}\left(x_{n}\right)}+\|\eta\|_{L_{\delta}^{p}\left(X_{n}\right)} .
$$

Since $A^{\prime}$ is irreducible by the unique continuation theorem [7, Lemma (4.3.2)], we have an elliptic estimate 


$$
\|\chi\|_{C 0\left(X_{n}\right)} \leqq c_{n}\left\|d_{A^{\prime}} \chi\right\|_{L^{q}\left(X_{n}\right)} \leqq c_{n}\left\|d_{A^{\prime}} \chi\right\|_{L}^{q q / p}\left(X_{n}\right)
$$

for some constant $c_{n}>0$ (c.f. [7, (7.2.30)]). So $B_{1}$ is a norm. Let $B_{2}$ be the completion of $\Omega_{X_{n}}^{1}\left(\operatorname{Ad} P_{n}\right)$ in the norm:

$$
\|\alpha\|_{B_{2}}=\|\alpha\|_{L_{q \delta / p}^{q}\left(X_{n}\right)}+\left\|d_{A^{\prime}}^{+} \alpha\right\|_{L_{\delta}^{p}\left(X_{n}\right)} .
$$

Then $M$ can be extended to a map from $B_{1}$ to $B_{2}$ and the derivative at $(0,0)$ is given by

$$
T(\chi, \eta)=D M_{(0,0)}(\chi, \eta)=d_{A^{\prime}} \chi+P \eta .
$$

By definition, $\mathrm{T}$ is a bounded map from $B_{1}$ to $B_{2}$.

LEMMA 5.8. There is a larger $n_{0} \in \boldsymbol{N}$ such that if $n>n_{0}$ then

$$
\|(\chi, \eta)\|_{B_{1}} \leqq 4\|T(\chi, \eta)\|_{B_{2}} .
$$

Proof. Let $\alpha=d_{A^{\prime}} \chi+P \eta$, so that $\mathrm{rfJ} » \alpha=[$ FJs $\chi]+\eta$. Since

$$
d_{\pi^{*} \Gamma} \chi=d_{A^{\prime}} \chi+\gamma_{i}\left[\left(A_{i}-\pi^{*} \Gamma\right), \chi\right]
$$

on $\tau_{\imath}^{-1}([n-2, n-1])$, we have an elliptic estimate

$$
\begin{aligned}
& \|\chi\|_{C 0\left(\tau_{n}^{-1}([n-2, n-1])\right)} \\
& \leqq c\left\|\left.d_{\pi^{*} \Gamma} \chi\right|_{\tau_{n}^{-1}([n-2, n-1])}\right\|_{L^{q}\left(X_{n}\right)} \\
& \leqq c\left\|\left.d_{A^{\prime}} \chi\right|_{\tau_{n}^{-1}([n-2, n-1])}\right\|_{L^{q}\left(X_{n}\right)}+c e^{-\lambda n}\|\chi\|_{C 0\left(\tau_{n}^{-1}([n-2, n-1])\right)} .
\end{aligned}
$$

For $n>n_{0}$ with $c e^{-\lambda n_{0}}<1 / 2$, we can rearrange this to get

$$
\|\chi\|_{C 0\left(\tau_{n}^{-1}([n-2, n-1])\right)} \leqq 2 c\left\|\left.d_{A^{\prime}} \chi\right|_{\tau_{n}^{-1}([n-2, n-1])}\right\|_{L^{q}\left(X_{n}\right)} .
$$

Noting that the support of $\left[F_{A^{\prime}}^{+}, \chi\right]$ is contained in $\tau_{n}^{-1}([n-2, n-1])$, we see that

$$
\begin{aligned}
&\|\eta\|_{L_{\delta}^{p}\left(X_{n}\right)} \leqq\|\alpha\|_{B_{2}}+\left\|\left[F_{A^{\prime}}^{+}, \chi\right]\right\|_{L_{\delta}^{p}\left(X_{n}\right)} \\
& \\
& \leqq\|\alpha\|_{B_{2}}+\left\|F_{A^{\prime}}^{+}\right\|_{L_{\delta}^{p}\left(X_{n}\right)}\|\chi\|_{C 0\left(\tau_{n}^{-1}([n-2, n-1])\right)} \\
& \leqq\|\alpha\|_{B_{2}}+c e^{-(\lambda-\delta / p) n}\left\|\left.d_{A^{\prime}} \chi\right|_{\tau_{n}^{-1}([n-2, n-1])}\right\|_{L_{q \delta / p}^{q}\left(X_{r}\right)} \\
& \leqq\|\alpha\|_{B_{2}}+c e^{-(\lambda-\delta / p) n}\|\alpha-P \eta\|_{L_{q \delta / p}^{q}\left(X_{n}\right)} \\
& \leqq\|\alpha\|_{B_{2}}+c e^{-(\lambda-\delta / p) n}\left(\|\alpha\|_{B_{2}}+\|\eta\|_{L_{\delta}^{p}\left(X_{n}\right)}\right) .
\end{aligned}
$$

Thus, for $n>n_{0}$ with $c e^{-(\lambda-\delta / p) n_{0}}<1 / 2$, we obtain

$$
\|\eta\|_{L}^{p}\left(X_{n}\right) \leqq 3\|\alpha\|_{B_{2}}
$$

This gives us then

$$
\|(\chi, \eta)\|_{B_{1}} \leqq 4\|\alpha\|_{B_{2}} .
$$


On the other hand, the operator $P$ is a pseudo-differential operator and the symbol is homotopic to that of $\left(d_{A^{\prime}}^{+}\right)^{*}\left(1+\Delta_{A^{\prime}}\right)^{-1}$. It follows that $d_{A^{\prime}}+P$ is Fredholm and its index equals that of $d_{A^{\prime}}+\left(d_{A^{\prime}}^{+}\right)^{*}$. So

$$
\operatorname{index}(T)=2 l_{X_{n}}+3\left(1+b_{+}\left(X_{n}\right)\right)=0 \text {. }
$$

Thus $T$ is an isomorphism from $B_{1}$ to $B_{2}$ with operator norm $\left\|T^{-1}\right\| \leqq 4$. This implies that $M$ is invertible near $(0,0)$ and 5 is open. It follows that $S=[0,1]$ and the proof is completed. D

COROLLARY 5.9. For $0<\boldsymbol{\nu}<\nu_{0}$ and $n>n_{0}(\nu)$, the intersection $U(\nu) \cap \mathscr{M}_{X_{n}}\left(l_{X_{n}}\right.$, $\left.\eta, g_{n}\right)$ is equal to the image of $I_{n}$.

Suppose that $I_{n}$ is not surjective for any large $n$. Then there are a subsequence $\{n\}$ (now we relabeled) with $n \rightarrow \infty$ and a sequence of $g_{n}$-ASD connections $\left\{A_{n}\right\}$ not coming from the map $I_{n}$. Uhlenbeck's compactness principle ([22], $[7,4.4])$ and the preservation of $w_{2}(P)$ under weak limit imply that, after taking a subsequence, the following data exists:

(1) A bundle $P^{\prime} \rightarrow Y_{1} \amalg Y_{2}$ with $w_{2}\left(\left.P^{\prime}\right|_{Y_{1}}\right)=\sigma_{1}^{*}(\eta), w_{2}\left(\left.P^{\prime}\right|_{Y_{2}}\right)=\sigma_{2}^{*}(\eta)$,

(2) ASD connections $A_{1}$ on $\left.P^{\prime}\right|_{Y_{1}}$ and $A_{2}$ on $\left.P^{\prime}\right|_{Y_{2}}$ with $\left(-1 / 4 \pi^{2}\right) \int_{J X_{1}} \operatorname{Tr}\left(F_{A_{1}}\right.$ $\left.\wedge F_{A_{1}}\right)=l_{1}$ and $\left(-1 / 4 \pi^{2}\right) \int_{Y_{2}} \operatorname{Tr}\left(F_{A_{2}} \wedge F_{A_{2}}\right)=l_{2}$,

(3) A collection of points $\left\{x_{1}, \cdots, x_{a}\right\} \in Y_{1},\left\{x_{a+1}, \cdots, x_{a+b}\right\} \in Y_{2}$,

(4) $C^{\infty}$-gauge transformations $\left\{k_{n}\right\}$ over $X_{n} \backslash\left\{x_{1}, \cdots, x_{a+b}\right\}$,

(5) fen/L^ converges to $A_{1}, A_{2}$ in $C^{\infty}$ on compact subsets of $Y_{1} \backslash\left\{x_{1}, \cdots, x_{a}\right\}$ III $Y_{2} \backslash\left\{x_{a+1}, \cdots, x_{a+b}\right\}$,

(6) $4 a+4 b-l_{1}-l_{2} \leqq-l_{X_{n}}$.

Since $\sigma_{1}^{*}(\eta) \neq 0$ and $\sigma_{2}^{*}(\eta) \neq 0$, there are no flat connections on $\left.P^{\prime}\right|_{Y_{1}}, P^{\prime}{ }_{Y_{2}}$, which implies that $l_{1}<0$ and $l_{2}<0$. Lemma 5.1 and Lemma 3.5 supply $h_{i} \in C^{\infty}$ (Aut $P$ ) such that $\left[h_{\imath} A_{\imath}\right]$ lies in $\mathscr{M}_{Y}\left(l_{\imath}, \sigma_{\imath}^{*}(\eta), g_{\imath}\right)$. By Proposition 3.7, we obtain $l_{1}=l_{Y_{2}} l_{2}=l_{Y_{\alpha}}$ and $a=b=0$.

LEMMA 5.10. There are constants $\varepsilon>0, t_{0}=t_{0, m}>0$ and $\rho=\rho_{m}>0$, independent of $T$, with the following significance Let $A$ be an ASD connection on $Q \times$ $(-T, \mathrm{~T})$ with $\int_{Z \times(-T, T)}\left|F_{A}\right|^{2}<\varepsilon$. Then there exists $h_{T} \in C^{\infty}(\operatorname{Aut}(Q \times(-T+1, T-$ 1)) such that for $-T+t_{0} \leqq t \leqq T-t_{0}$,

$$
\begin{gathered}
\sup _{z \times[t-1, t+1]}\left\{\sum_{t=0}\left|\nabla_{\pi^{*}+T^{\prime}}\left(h_{\Gamma^{r}} A-\pi^{*} \Gamma\right)\right|^{2}\right\} \leqq \rho \rho^{-2 \lambda(T-|t|)}, \\
i_{\partial / \partial t}\left(h_{T} A-\pi^{*} \Gamma\right)=0 .
\end{gathered}
$$

Proof. The proof is very similar to that of Lemma 5.1. We apply Proposition 4.2 over $Z \times(-T, T)$ to obtain a gauge transformation $h \in C^{\infty}(\operatorname{Aut}(Q \mathrm{X}$ $[-\mathrm{T}+1, T-1]))$ such that for $-T+8 \leqq t \leqq T-8$, 


$$
\sup _{Z \times[t-1, t+1]}\left\{\sum_{i=0}^{m}\left|\nabla_{\pi^{*}}^{(l)}\left(h A-\pi^{*} \Gamma\right)\right|^{2}\right\}<c e^{-2 \lambda(T-|t|)} .
$$

Solve the ordinary differential equation for $\tilde{h} \in C^{\infty}(\operatorname{Aut}(Q \times[-T+1, T-1]))$ :

$$
i_{\partial / \partial t}\left(\tilde{h}(h A)-\pi^{*} \Gamma\right)=0
$$

with initial condition $\left.\tilde{h}\right|_{t=0}=1$. Then by (5.1) we have

$$
\frac{\partial}{\partial t}\left|d_{\Gamma} \tilde{h}\right|^{2} \leqq c e^{-\lambda(T-|t|)}\left(\left|d_{\Gamma} \tilde{h}\right|^{2}+\left|d_{\Gamma} \tilde{h}\right|\right) .
$$

If $t \geqq 0$, then we integrate over $[0, t+1]$ to get an inequality

$$
\left\|d_{\Gamma} \tilde{h}\right\|_{C 0(Z \times[0, t+1])}^{2} \leqq c e^{-\lambda(T-t)}\left(\left\|d_{\Gamma} \tilde{h}\right\|_{C 0(Z \times[0, t+1])}^{2}+\left\|d_{\Gamma} \tilde{h}\right\|_{C 0(Z \times[0, t+1])}\right) .
$$

For $t_{0}>0$ with $c e^{-\lambda t_{0}}<1 / 2$ and $0 \leqq t \leqq T-t_{0}-1$, we obtain

$$
\left\|d_{\Gamma} \tilde{h}\right\|_{C 0(Z \times[t-1, t+1])} \leqq\left\|d_{\Gamma} \tilde{h}\right\|_{C 0(Z \times[0, t+1])} \leqq 2 c e^{-\lambda(T-\mid t))} .
$$

In the case $t \leqq 0$, we can also prove the above by the parallel discussion. So by bootstrapping (5.2), we see that $h_{T}=\tilde{h} h$ satisfies the desired properties for some $\rho>0$. D

$W e$ choose $n_{0} \in \boldsymbol{N}$ so that

$$
\int_{\imath_{\imath}^{-1}\left(\left[n_{0}, \infty\right)\right)}\left|F_{A_{\imath}}\right|^{2}<\frac{1}{2} \varepsilon
$$

Since

$$
\lim _{n \rightarrow \infty} \int_{\tau_{n}^{-1}\left(\left[n_{0}, n\right]\right)}\left|F_{A_{n}}\right|^{2}=\int_{\tau_{n}^{-1}\left(\left[n_{0}, \infty\right)\right)}\left|F_{A_{1}}\right|^{2}+\int_{\tau_{n}^{-1}\left(\left[n_{0}, \infty\right)\right)}\left|F_{A_{2}}\right|^{2},
$$

we have

$$
\int_{\tau_{n}^{-1}\left(\left[n_{q}, n\right]\right)}\left|F_{A_{n}}\right|^{2}<\varepsilon\left(n>n_{1}\right)
$$

for some $n_{1}>n_{0}$. By Lemma 5.10, we can find $h_{n} \in C^{\infty}\left(\right.$ Aut $\left(Q \times\left[n_{0}+1, n+1\right] \cup_{\widetilde{\psi}_{n}}\right.$ $\left.Q \times\left[n_{0}+1, n+1\right]\right)$ such that for $n_{0}+t_{0} \leqq t \leqq n$,

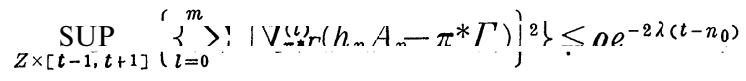

$$
\begin{aligned}
& i_{\partial \jmath \partial t}\left(h_{n} A_{n}-\pi^{*} \Gamma\right)=0 .
\end{aligned}
$$

So the connection $\left(h_{n} A_{n}\right)^{\prime}=\gamma_{i}\left(h_{n} A_{n}-\pi^{*} \Gamma\right)+\left(1-\gamma_{i}\right) \pi^{*} b \operatorname{ver} \tau_{\imath}^{-1}\left(\left[n_{0}+1, \infty\right)\right)$ also satisfies the same condition as above for $n_{0}+t_{0} \leqq t \leqq n-1$. Now we can apply Ascoli-Arzela's theorem with diagonal argument to deduce that, after taking a subsequence, $\left(h_{n} A_{n}\right)^{\prime}$ converges to an ASD connection $A_{\imath}^{\prime \prime}$ in $C^{m-1}$ over compact sets in $\tau_{2}^{-1}\left(\left[n_{0}+t_{0}, \infty\right)\right)$. So $A^{\prime} l$ satisfies 


$$
\begin{gathered}
\operatorname{sip}_{i}^{-1}([t-1, t+1])\left(\sum_{l=0}^{i m-1}\left|\nabla_{\pi^{*}}^{(l)}\left(A_{\imath}^{\prime \prime}-\pi^{*} \Gamma\right)\right|^{2}\right\} \leqq c_{i} e^{-2 \lambda\left(t-n_{0}\right)}, \\
i_{\partial / \partial t}\left(A_{\imath}^{\prime \prime}-\pi^{*} \Gamma\right)=0
\end{gathered}
$$

for $t \geqq n_{0}+t_{0} \quad$ By Lebesgue convergence theorem we have

$$
\left\|\left.\left(\left(h_{n} A_{n}\right)^{\prime}-A_{\imath}^{\prime \prime}\right)\right|_{\tau_{n}^{-1}\left(\left[n_{0}+t_{0}, \infty\right)\right)}\right\|_{L_{q}^{q} \delta / p}\left(Y_{i}\right) \longrightarrow 0(n \rightarrow \infty) .
$$

We bootstrap the equation

$$
d_{A_{i}}\left(h_{n} k_{n}^{-1}\right)=h_{n} k_{n}^{-1}\left(k_{n} A_{n}-A_{\imath}\right)-\left(h_{n} A_{n}-A_{\imath}\right) h_{n} k_{n}^{-1},
$$

to deduce that there is a subsequence $\left\{h_{n} k_{n}^{-1}\right\}$ (now we relabeled) such that $h_{n} k_{n}^{-1}$ converges to some $u_{\imath}$ in $C^{m}$ on $\tau_{\imath}^{-1}\left(\left(n_{0}+t_{0}, n_{0}+t_{0}+1\right)\right)$ By Lemma 3.5, if we replace $\left\{h_{n}\right\}$ by $\left\{r_{i} h_{n}\right\}$ for some $r_{i} \in \mathscr{R}$, we can suppose that $\tilde{u}_{\imath}$ can be extended to $u^{*}$ over $\tau_{\imath}^{-1}\left(\left[0, n_{0}+t_{0}+1\right)\right)$ and $\mathrm{b}\left(1, \mathrm{fi}_{\mathrm{t}}\right)=0$. Then for large $n \in \boldsymbol{N}$, $\mathrm{b}\left(1, r_{i} h_{n} k_{n}^{-1}\right)=\mathrm{b}\left(1, \tilde{u}_{\imath}\right)=0$. So we have $\mathrm{b}\left(1, r_{2} r_{1}^{-1}\right)=\mathrm{b}\left(1, r_{2} h_{n} k_{n}^{-1}\left(r_{1} h_{n} k_{n}^{-1}\right)^{-1}\right)=$ $\mathrm{b}\left(1, r_{2} h_{n} k_{n}^{-1}\right)+\delta\left(1, r_{1} h_{n} k_{n}^{-1}\right)=0$. Since $\left(r_{2} r_{1}^{-1}\right) \Gamma=\Gamma$,we see that $r_{1}=r_{2}$. Now we can apply the argument of [7, Lemma (4.4.5)] (see also Appendix 1) to patch gauge transformations $k_{n}$ and $r_{i} h_{n}$ over $\tau_{2}^{-1}\left(\left(n_{0}+t_{0}, n_{0}+t_{0}+1\right)\right)$ Then taking a subsequence, we can find $C^{m-1}$ gauge transformations $\left\{u_{n}\right\}$ on $X_{n}$ such that $u_{n}=h_{n}$ on $\tau_{n}^{-1}\left(\left[n_{0}+t_{0}+1, n\right]\right)$ and $u_{n} A_{n}$ converges to an ASD connection $\hat{A}_{\imath}$ on $P_{\imath}$ in $C^{m-2}$ over compact subsets of $Y_{\imath}$, which is in the exponential gauge. If we choose $m \geqq 5$, then $\tilde{A}_{\imath}$ is gauge equivalent to a $C^{\infty}$-connection and the uniqueness in Lemma 5.1 implies that $\tilde{A}_{\imath}$ is smooth over $Z \times\left[n_{0}+t_{0}, \infty\right)$ and satisfies the condition (ii) for $t \geqq n_{0}+t_{0}$. So we obtain

$$
\left\|u_{n} A_{n}-J_{n}\left(\tilde{A}_{1} A_{2}\right)\right\|_{L_{\sigma_{i} \zeta n_{n}}^{q}\left(X_{n}\right)} \longrightarrow 0(n \rightarrow \infty) .
$$

Now by Corollary 5.9, $\left[A_{n}\right]-\left[I_{n}\left(A_{1}, \tilde{A}_{2}\right)\right] \in \mathcal{M}_{X_{n}}\left(l_{X_{n}} \eta, g_{n}\right)$ for large $n$, which is a contradiction.

Next for $n \in N$, we consider the oriented 4 -manifold $K_{i n}^{*}$ defined by

$$
K_{i n}^{*}=K_{i} \cup Z \times[0, n+1] \bigcup_{\phi_{i n}} Z \times[0, n+1] \cup W,
$$

and the $S O(3)$ bundle $P_{\imath n}$ over $K_{\imath n}^{*}$ defined by

$$
P_{\imath n}=P_{0 i} \bigcup_{i i} Q \times[0, n+1] \widetilde{\boldsymbol{\phi}}_{i n} Q \times[0, n+1] \cup \tilde{Q}
$$

for each $\imath-1,2$. Here $\phi_{i n}$ and $\ddot{\phi}_{i n}$ are defined by using $\phi_{i}$ and a lift $\tilde{\phi}_{i}: Q \rightarrow$ $Q$ with $\tilde{\phi}_{i} \Gamma=\Gamma$ as before. Let $\mathcal{C}_{K_{\imath n}^{*}}^{r}(r \geqq 3)$ be the space of all conformal classes of $C^{r}$-metrics on $K_{i n}^{*}$ which are a fixed metric on $W$ and $h+d t^{2}$ on $Z \times[0, n$ $+1] \cup_{\phi_{\imath n}} Z \times[0, n+1]$. If we fix a metric $\left[g_{i 0}\right]$ in $\mathcal{C}_{K_{\imath n}}^{r}$, then $\mathcal{C}_{K_{\imath n}}^{r}$ is identified with

$$
\left\{m: \Lambda^{+} \rightarrow \Lambda^{-} ; C^{r} \text {-bundle map, } \sup |m|<1,\left.m\right|_{\left.K_{\imath n}^{*} \backslash K_{i}^{0}=0\right\}},\right.
$$


where $\Lambda^{ \pm}$is \pm self-dual space with respect to $g_{i 0}$. Then we have the following theorem, whose proof is also the same as in ([7, 4.3], [14, 5 (iii)]).

PROPOSITION 5.11. There is a Baire set $\mathcal{C}_{K_{\imath n}^{*}}^{\prime *} \subset \mathcal{C}_{K_{\imath n}}^{*}$ such that for all $g \in$ $\mathcal{C}_{K_{\imath n}^{*}}^{*}$, the $\Lambda S D$ moduli $\mathscr{M}_{K_{i n}^{*}}^{*}\left(l_{K_{i n}}^{*}, \eta_{\imath}^{*}, g\right)$ is a finite set consisting of irreducible regular connections.

For each $n \in \boldsymbol{N}$, we fix a metric $g_{\imath n}$ in $\mathcal{C}_{K_{\imath n}^{*}}^{*}$ such that $g_{\imath n}$ is independent of $n$ on $K_{2}^{0}$ and the metric

$$
g_{\imath}= \begin{cases}g_{\imath n} & \text { on } K_{\imath}, \\ h+d t^{2} & \text { on } \mathrm{Zx}[0, \infty),\end{cases}
$$

lies in $\mathcal{C}_{Y_{\imath}}^{\prime}$ for $\imath=1,2$.

$W$ extend $Q$ and $f$ naturally over $W \cup Z \times[0,00)$, which we also denote by $\tilde{\Gamma}$ and $\tilde{Q}$ respectively. We replace $Y_{2}$ by $W \cup Z \times[0$, oo) and set a function $\tau_{2}: Y_{2} \rightarrow \boldsymbol{R}$ as before. Then we can define the gluing map

$$
I_{1 n}: \mathscr{M}_{Y_{1}}\left(l_{Y_{1}}, \sigma_{1}^{*}(\eta), g_{1}\right) \times\{\tilde{\Gamma}\} \longrightarrow \mathscr{M}_{K_{1 n}^{*}}^{*}\left(l_{K_{1 n}^{*}}, \eta_{1}^{*}, g_{1 n}\right)
$$

for large $n \in \boldsymbol{N}$ just as before. We can prove that $I_{1 n}$ is bijective in the same way as that of $/$. But in the proof, we need to correct it at some points. First, after Corollary 5.9, we treat a sequence of connections $\left\{A_{n}\right\}$. Then we obtain $l_{1}=l_{Y_{1}}, l_{2}=0$ and $a=b=0$. So $u_{2}^{*} A_{2}=\tilde{\Gamma}$ for some $C^{\infty}$-gauge $u_{2}^{*}$ on $W \cup Z$ $\times\left[0\right.$, oo) and $u_{2}^{*} k_{n} A_{n}$ converges to $\tilde{\Gamma}$ in $C^{\infty}$ on compact subsets of $W \cup Z \times$ [0, oo). Second, after (5.6), on $\tau_{1}^{-1}\left(\left(n_{0}+t_{0}, n_{0}+t_{0}+1\right)\right)$, if we replace $\left\{h_{n}\right\}$ by $\left\{r_{1} h_{n}\right\}$ for some $r_{1} \in \mathcal{R}$, we can suppose that $r_{1} h_{n} k_{n}^{-1}$ converges to $u_{1}$ over $\tau_{1}^{-1}\left(\left(n_{0}+t_{0}, n_{0}+t_{0}+1\right)\right) \quad W$ e bootstrap the equation

$$
d_{\pi^{*} \Gamma}\left(r_{1} h_{n}\left(u_{2}^{*} k_{n}\right)^{-1}\right)=r_{1} h_{n}\left(u_{2}^{*} k_{n}\right)^{-1}\left(u_{2}^{*} k_{n} A_{n}-\pi^{*} \Gamma\right)-r_{1}\left(h_{n} A_{n}-\pi^{*} \Gamma\right) h_{n}\left(u_{2}^{*} k_{n}\right)^{-1}
$$

to obtain that, if we choose $n_{0}$ so large, then there is a subsequence $\left\{r_{1} h_{n}\left(u_{2}^{*} k_{n}\right)^{-1}\right\}$ (now we relabeled) such that $r_{1} h_{n}\left(u_{2}^{*} k_{n}\right)^{-1}$ converges to some $u_{2}$ in $C^{m-1}$ on $\tau_{2}^{-1}\left(\left(n_{0}+t_{0}, n_{0}+t_{0}+1\right)\right)$ and, since the right hand side is so small, $u_{2}$ can be extended to $\tilde{u}_{2}^{*}$ over $\tau_{2}^{-1}\left(\left[0, n_{0}+t+1\right)\right)$. Now we can apply the argument of [7, Lemma (4.4.5)] to patch gauge transformations $k_{n}$ (resp. $u_{2}^{*} k_{n}$ ) and $r_{1} h_{n}$ over $\tau_{1}^{-1}\left(\left(n_{0}+t_{0}, n_{0}+t_{0}+1\right)\right)$ (resp. $\left.\tau_{2}^{-1}\left(\left(n_{0}+t_{0}, n_{0}+t_{0}+1\right)\right)\right)$. Then as was shown there, we see that $I_{1 n}$ is surjective for large $n \in N$. (This bijection has been stated in [10, Theorem V. 3.4].) Of course, the same holds for $Y_{2}$ and $I_{2 n}$ defined as before. Composing three bijections, we have a bijection

$$
K_{n}: \mathscr{M}_{K_{1 n}^{*}}\left(l_{K_{1 n}^{*}}, \eta_{1}^{*}, g_{1 n}\right) \times \mathscr{M}_{K_{2 n}^{*}}^{*}\left(l_{K_{2 n}^{*}}^{*}, \eta_{2}^{*}, g_{2 n}\right) \longrightarrow \mathscr{M}_{X_{n}}\left(l_{X_{n}}, \eta, g_{n}\right)
$$

for large $n \in \boldsymbol{N}$. Hence Theorem 2.1 follows from the lemma below.

LEMMA 5.12. If $\left[B_{i 0}\right],\left[B_{i 1}\right] \in \mathscr{M}_{K_{\imath n}^{*}}^{*}\left(l_{K_{\imath n}^{*}}, \eta_{i}^{*}, g_{\imath n}\right)$ satisfies $o\left(\left[B_{i 1}\right]\right)=\varepsilon_{i} o\left(\left[B_{i 0}\right]\right)$ $(i=1,2)$, then $o\left(K_{n}\left(\left[B_{11}\right],\left[B_{21}\right]\right)\right)=\varepsilon_{1} \varepsilon_{2} o\left(K_{n}\left(\left[B_{10}\right],\left[B_{20}\right]\right)\right)$. 
Proof. We write $\left[B_{i 0}\right]=\left[I_{\imath n}\left(A_{i 0}, \tilde{\Gamma}\right)\right],\left[B_{i 1}\right]=\left[I_{\imath n}\left(A_{i 1}, \mathrm{f}\right)\right]$ for some $\left[A_{i 0}\right]$, $\left[A_{i 1}\right] \in \mathscr{M}_{Y_{i}}\left(l_{Y_{i}}, \sigma_{i}^{*}(\eta), g_{\imath}\right)$. We choose any path $A_{i t}(0 \leqq t \leqq 1)$ in $\mathcal{A}_{P_{\imath}}$ from $A_{i 0}$ to $A_{i 1}$. Define a connection $A_{i t}^{\prime}$ on $P_{\imath n}$ by

$$
A_{i t}^{\prime}=\left\{\begin{array}{lll}
\mu_{i}\left(A_{i t}-\pi^{*} \Gamma\right)+\left(1-\mu_{i}\right) \pi^{*} \Gamma & \text { over } & \tau_{i}^{-1}([0, n+1]), \\
\tilde{\Gamma} & \text { over } & W \cup Z \times[0, n+1],
\end{array}\right.
$$

and a connection $A_{t}^{\prime}$ on $P_{n}$ by $A_{t}^{\prime}=J_{n}\left(A_{1 t}, A_{2 t}\right)$. Since $\left[A_{i_{0}}^{\prime}\right]$ (resp. [ $\left.A_{i 1}\right]$ ) lies in $U\left(\left[A_{i 0}\right]\right)$ (resp. $U\left(\left[A_{i 0}\right]\right)$ ) for large $n \in N$, we have a nowhere zero section $s_{\imath}$ over $\left[A_{i t}^{\prime}\right](0 \leqq t \leqq 1)$ such that

$$
s_{i}\left(\left[A_{i 0}^{\prime}\right]\right)=\varepsilon_{i} o\left(\left[B_{i 0}\right]\right), \quad s_{i}\left(\left[A_{i 1}^{\prime}\right]\right)=o\left(\left[B_{i 1}\right]\right) .
$$

Since $\operatorname{Ker} \delta_{A_{i t}^{\prime}}^{*}$ is supported in $\tau_{\imath}^{-1}([0, n-1])$, we can choose a linear map $S_{\imath}: \boldsymbol{R}^{N_{\imath}} \rightarrow$ $L_{2}^{2}\left(\left(\Omega_{K_{i n}^{*}}^{0} \oplus \Omega_{K_{\imath n}^{*}}^{+}\right)\left(\operatorname{Ad} P_{\imath n}\right)\right)$ so that for all $0 \leqq t \leqq 1, \delta_{A_{i t}^{\prime}} \oplus S_{\imath}$ is surjective and the image of $S_{\imath}$ is supported in $\tau_{\imath}^{-1}([0, n-1])$. So $\operatorname{Ker}\left(\delta_{A_{i t}^{\prime}} \oplus S_{\imath}\right)$ is supported in $\tau_{\imath}^{-1}([0, n-1]) \times \boldsymbol{R}^{N_{\imath}}(i=1,2)$ and $\operatorname{Ker}\left(\delta_{A_{t}^{\prime}} \oplus S_{1} \oplus S_{2}\right)$ is supported in $\tau_{n}^{-1}([0, n-1]) \times$ $\boldsymbol{R}^{N_{1}+N_{2}}$. Then we have a natural isomorphism

$$
\begin{gathered}
\theta_{t}: \operatorname{Ker}\left(\delta_{A_{1 t}^{\prime}} \oplus S_{1}\right) \oplus \operatorname{Ker}\left(\delta_{A_{2 t}^{\prime}} \oplus S_{2}\right) \longrightarrow \operatorname{Ker}\left(\delta_{A_{t}^{\prime}} \oplus S_{1} \oplus S_{2}\right) \\
\left(t_{1}, t_{2}\right) 1 \longrightarrow t_{1}+t_{2},
\end{gathered}
$$

by which we obtain a nowhere zero section $\left(\Lambda^{\max } \theta_{t}\right)\left(s_{1} \otimes s_{2}\right)$ on $\Lambda_{P_{n}}$ over $\left[A_{t}^{\prime}\right]$ $(0 \leqq t \leqq 1)$. Since $\operatorname{Ker} \delta_{A_{t}^{\prime}}^{*_{1}}$ is supported in $\tau_{n}^{-1}([0, n-1]), \delta_{A} \oplus S_{1} \oplus S_{2}$ is surjective. Now we get

$$
\begin{aligned}
& \left(\Lambda^{\max } \theta_{0}\right)\left(s_{1} \otimes s_{2}\right)\left(\left[A_{0}^{\prime}\right]\right)=\left(\Lambda^{\max } \theta_{0}\right)\left(s_{1}\left(\left[A_{10}^{\prime}\right]\right) \otimes s_{2}\left(\left[A_{20}^{\prime}\right]\right)\right) \\
& =\varepsilon_{1} \varepsilon_{2}\left(\Lambda^{\max } \theta_{0}\right)\left(o\left(\left[B_{10}\right]\right) \otimes o\left(\left[B_{20}\right]\right)\right)=\varepsilon_{1} \varepsilon_{2} o\left(\left[I_{n}\left(A_{10}, A_{20}\right)\right]\right) \\
& =\varepsilon_{1} \varepsilon_{2} o\left(K_{n}\left(\left[B_{10}\right],\left[B_{20}\right]\right)\right), \\
& \left(\Lambda^{\max } \theta_{1}\right)\left(s_{1} \otimes s_{2}\right)\left(\left[A_{1}^{\prime}\right]\right)=o\left(K_{n}\left(\left[B_{11}\right],\left[B_{21}\right]\right)\right) .
\end{aligned}
$$

This implies the desired equality.

Proof of Theorem 2.2. Suppose that $\gamma_{X}(\eta) \neq 0$. Then for each $n$ there is an ASD connection $\left[A_{n}\right]$ in $\mathscr{M}_{X_{n}}\left(l_{X_{n}}, \eta, g_{n}\right)$. (Here, in the construction of $P_{n}, \iota_{1}$ and $\iota_{2}$ are chosen so that $w_{2}\left(P_{n}\right)=\eta, p_{1}\left(P_{n}\right)=l_{K_{n}}$ are only satisfied.) Then after taking a subsequence, we have the data from (1) to (6) in the above. As was shown there, we see that $l_{1}=l_{Y_{1}}$ and $l_{2}=l_{Y_{2}}$. The resulting connection $A_{2}$ on $P_{\imath}$ can be thought of an element in $\mathscr{M}_{Y_{i}}\left(l_{Y_{i}}, \boldsymbol{\sigma}_{i}(\eta), g_{\imath}\right)$. The following lemma contradicts to the assumption. $\Pi$

LEMMA 5.13. For any connection $A$ on $P$ which is isomorphic to $\pi^{*} \Gamma$ on $Z \times[n, \infty)$ for some $n \in \boldsymbol{N}$, 


$$
\frac{-1}{4 \pi^{2}} \int_{Y} \operatorname{Tr}\left(F_{A} \wedge F_{A}\right) \equiv\left(\eta^{*}\right)^{2} \quad(\bmod 2) .
$$

Proof. Define $\tilde{P}=\left.P\right|_{\tau^{-1}([0, n])} \cup \tilde{Q} . \quad \sigma^{*}\left(w_{2}(\tilde{P})\right)=\sigma^{*}\left(\eta^{*}\right)$ implies that $w_{2}(\tilde{P})=\eta^{*}$ or $w_{2}(\widetilde{P})=\eta^{*}+$ P.D. $\left[T^{2} \times 0\right]$. In any case, we obtain

$$
\frac{-1}{4 \pi^{2}} \int_{Y} \operatorname{Tr}\left(F_{A} \wedge F_{A}\right)=p_{1}(\tilde{P}) \equiv\left(w_{2}(\tilde{P})\right)^{2} \equiv\left(\eta^{*}\right)(\bmod 2)
$$

Remark. The vanishing of $\gamma_{X}(\eta)$ can be observed for more general elements $\eta \in C_{X}$. In a forthcoming paper, we will treat it.

\section{Explicit calculations on elliptic surfaces.}

In this section we calculate values of the simple invariant for the regular elliptic surfaces without multiple fibers. Let $\pi: S_{k} \rightarrow \boldsymbol{C P} \boldsymbol{P}^{1}$ be a regular minimal elliptic surface with $p_{g}=k-l$ and without multiple fibers. Then $S_{k}$ satisfies $\pi_{1}\left(S_{k}\right)=1, b_{+}\left(S_{k}\right)=2 k-1$ and $l_{S_{k}-}-3 k$. It admits a differentiable section $\Sigma_{k}$. $\boldsymbol{C} \boldsymbol{P}^{1} \rightarrow S_{k}$, which has the self-intersection number $\left(\Sigma_{k}\right)^{2}=-k$. We take a general fiber / in $S_{k}$. Then we can interpret this surface $S_{k}$ as a fiber sum up to fiber preserving diffeomorphism as follows [17]: Given $S_{\imath}$ and $S_{k-i}(1 \leqq i \leqq k-1)$, identify the tubular neighborhood of a general fiber in each with $T^{2} \times D^{2}$ so that the fibrations correspond to projection onto $D^{2}$. Remove the interior of tubular neighborhoods from $S_{\imath}$ and $S_{k-\imath}$, and glue the two remaining manifolds together by an orientation reversing and fiber preserving diffeomorphism on the boundaries. Then we get an oriented manifold $S_{i} \natural S_{k-\imath}$, a fibration $\pi$ : $S_{i} \natural S_{k-i} \rightarrow \boldsymbol{C P} \boldsymbol{P}^{1}$, and a section $\sum_{i} \natural \sum_{k-\imath}: \boldsymbol{C P} \boldsymbol{P}^{1} \rightarrow S_{i} \natural S_{k-\imath}$. We note that $S_{2}$ contains the Kummer surface, which is one of the K3 surface.

We use a well known result by Donaldson ([6], [7], [13]).

PROPOSITION 6.1. (Donaldson) If we fix the orientation of $\mathrm{H}^{+}\left(\mathrm{S}_{2}\right)$ determined by the complex structure of $S_{2}$, then $\gamma_{S_{2}}(\eta)=1$ for any $\eta \in C_{S_{2}}$.

LEMMA 6.2. $\mid \gamma_{s_{3}}$ (P.D. $\left.\left(\left[\Sigma_{3}\right]+[f]\right)\right) \mid=1$.

Proof. Because $S_{6}=S_{3} \natural S_{3}=S_{2} \natural S_{2} \natural S_{2}$ and $\quad \Sigma_{6}=\Sigma_{3} \natural \Sigma_{3}=\Sigma_{2} \natural \Sigma_{2} \natural \Sigma_{2}: \boldsymbol{C P}^{1} \rightarrow$ $S_{6}$, we apply Theorem 2.1 three times to deduce that

$$
\begin{aligned}
\left|\gamma_{S_{6}}\left(\mathrm{P} . \mathrm{D} .\left[\Sigma_{6}\right]\right)\right| & \left.=\left|\gamma_{S_{6}}\left(\mathrm{P} . \mathrm{D} .\left(\left[\Sigma_{6}\right]+2[f]\right)\right)\right|=\mid \gamma_{S_{3}} \text { (P.D. }\left(\left[\Sigma_{3}\right]+[f]\right)\right)\left.\right|^{2} \\
& \left.=\mid \gamma_{S_{2}} \text { (P.D. }\left[\Sigma_{2}\right]\right)\left.\right|^{3}=1 .
\end{aligned}
$$

So $\left|\gamma_{s_{3}}\left(\operatorname{PD}\left(\left[\Sigma_{3}\right]+[f]\right)\right)\right|=1 . \mathrm{G}$

COROLLARY 6.3. For integer $k \geqq 2$,

$$
\left|\gamma_{s_{k}}\left(\mathrm{P} . \mathrm{D} .\left[\Sigma_{k}\right]\right)\right|=1 \quad \text { if } k \text { is even, }
$$




$$
\left.\mid \gamma_{s_{k}}\left(\mathrm{P} \text { D. }\left(\left[\Sigma_{k}\right]+[f]\right)\right)\right) \mid=1 \quad \text { if } k \text { is odd. }
$$

Remark. In a recent paper [11], we found that for integer $k \geqq 2$,

$$
\begin{gathered}
\left|\gamma_{s_{k}}\left(\mathrm{P} . \mathrm{D} \cdot\left[\Sigma_{k}\right]\right)\right| \neq 0 \quad \text { if } k \text { is even, } \\
\left|\gamma_{s_{k}}\left(\mathrm{P} . \mathrm{D} .\left(\left[\Sigma_{k}\right]+[/]\right)\right)\right| \neq 0 \quad \text { if } k \text { is odd. }
\end{gathered}
$$

using the moduli of stable vector bundles.

For $k$ odd, we can determine the image of $\left|\gamma_{s_{k}}\right|$.

LEMMA 6.4. $\left|\gamma_{S_{3}}(\eta)\right|=1$ for any $\eta \in C_{S_{3}}$.

Proof. Since the characteristic element is $w_{2}\left(S_{3}\right) \equiv$ P. D. $[f](\bmod 2),\langle\eta,[/]\rangle$ $\equiv \eta \eta \equiv 1(\bmod 2)$. We construct $\eta \sharp \eta \in C_{S_{6}}$, by identifying a tubular neighborhood of a general fiber in two copies of $S_{3}$. If we identify $S_{3}$ with $S_{2} S_{1}$, then $\eta \in$ $C_{S_{3}}$ factors as $\eta=\eta_{2} \natural \eta_{1}$ for some $\eta_{2} \in H^{2}\left(S_{2} ; \boldsymbol{Z}_{2}\right)$ and $\eta_{1} \in H^{2}\left(S_{1} ; \boldsymbol{Z}_{2}\right)$ with $\eta^{2} \equiv$ $\left(\eta_{2}\right)^{2}+\left(\eta_{1}\right)^{2}(\bmod 4)$. If $\left(\eta_{2}\right)^{2} \equiv 0(\bmod 4)$, then $\left(\eta_{2}+\text { P.D. }[f]\right)^{2} \equiv\left(\eta_{2}\right)^{2}+2 \equiv 2(\bmod 4)$. So we may assume that $\eta_{2} \in C_{S_{2}}, \eta_{1} \in C_{S_{1}}$. In the same way as above we have an element $\eta_{1} \eta_{1} \in C_{S_{2}}$. Now by Theorem 2.1 and Proposition 6.1, we get

$$
\left|\gamma_{s_{6}}(\eta \eta \eta)\right|=\left|\gamma_{s_{3}}(\eta)\right|^{2}=\left|\gamma_{s_{2}}\left(\eta_{2}\right)\left\|\gamma_{s_{2}}\left(\eta_{1} \eta \eta_{1}\right)\right\| \gamma_{s_{2}}\left(\eta_{2}\right)\right|=1 . \quad \mathrm{D}
$$

We can apply the above argument on $S_{k}=S_{2} \sharp S_{2} \sharp S_{3}$ to deduce that

COROLLARY 6.5. $/ / k$ is odd and $k \geqq 3$, then $\left|\gamma_{s_{k}}(\eta)\right|=$ for any $\eta \in C_{S_{k}}$. $1(\bmod 2)$.

COROLLARY 6.6. // $k$ is even, then $\left|\gamma_{s_{k}}(\eta)\right|=1$ for $\eta \in C_{S_{k}}$ with $\langle\eta,[f]\rangle \equiv$

Remark. In fact, Ue has obtained that the value $\left|\gamma_{S_{k}}(\eta)\right|$ is independent of $\eta \in C_{k}$ with $\langle\eta,[/]\rangle=1(\bmod 2)$, by analyzing the action of diffeomorphism group of $S_{k}$ on $C_{S_{k}}$ [21].

\section{Appendix 1}

Lemma (4.4.5) in [7] omits a necessary hypothesis on extension of gauge group. We write a precise statement, but omit its proof, since it is same as that of Lemma (4.4.5).

Lemma (4.4.5)'. We fix $m \in \boldsymbol{N}$. Suppose that $A_{n}$ is a sequence of unitary $C^{m}$-connections on a $S O(3)$ bundle $P$ over a base manifold $\Omega$ (possibly noncompact), and let $\tilde{\Omega} \Subset \Omega$ be an interior domain. Suppose that there are gauge transformations $u_{n} \in C^{m+1}$ (Aut $P$ ) and $\tilde{u}_{n} \in C^{m+1}$ (Aut $P \mid \tilde{\Omega}$ ) such that $u_{n} A_{n}$ converge in $C^{m}$ over $\Omega$ and $\tilde{u}_{n} A_{n}$ converges in $C^{m}$ over $\tilde{\Omega}$. Then we may assume that; taking a subsequence $\left\{n^{\prime}\right\}$, the $\tilde{u}_{n^{\prime}} u_{n^{\prime}}^{-1}$ converge in $C^{m}$ over $\tilde{\Omega}$ to a limit $\tilde{u}$. 
$/ / u$ extends over $\Omega$, then for any compact set $K \subset \tilde{\Omega}$ we can find gauge transformations $w_{n^{\prime}} \in C^{m}$ (Aut $P$ ) such that $w_{n^{\prime}}-\tilde{u}_{n^{\prime}}$ in a neighborhood of $K$ and the connections $w_{n} A_{n^{\prime}}$ converge in $C^{m-1}$ over $\Omega$.

In Section 5, we apply the above lemma with $\Omega=\tau_{2}^{-1}\left(\left[0, n_{0}+t_{0}+1\right)\right), \tilde{\Omega}=$ $\tau_{\imath}^{-1}\left(\left(n_{0}+t_{0}, n_{0}+t_{0}+(3 / 4)\right)\right)$ and $K=\tau_{\imath}^{-1}\left(\left[n_{0}+t_{0}+(1 / 4), n_{0}+t_{0}+(1 / 2)\right]\right)$.

\section{Appendix 2.}

We prove the compactness in Proposition 3.7. For $l_{Y}<l<0$, it is vacuous. Let $\left\{\left[A_{\imath}\right]\right\}$ be a sequence in $\mathscr{M}_{Y}\left(l_{Y}, \eta, g\right)$. Then after taking a subsequence, the following data exists :

(1) A bundle $P^{\prime} \rightarrow Y$ with $w_{2}\left(P^{\prime}\right)=\eta$,

(2) An ASD connection $A$ on $P^{\prime}$ with $\left(-1 / 4 \pi^{2}\right) \int_{Y} \operatorname{Tr}\left(F_{A} \wedge F_{A}\right)=l$,

(3) A collection of points $\left\{x_{1}, \cdots, x_{a}\right\} \in Y$,

(4) $C^{\infty}$-gauge transformations $\left\{k_{n}\right\}$ over $Y \backslash\left\{x_{1}, \cdots, x_{a}\right\}$,

(5) $k_{n} A_{n}$ converges to $A$ in $C^{\infty}$ on compact subsets of $Y \backslash\left\{x_{1}, \cdots, x_{a}\right\}$,

(6) $4 a-l \leqq-l_{Y}$.

Since $\eta \neq 0$, there are no flat connections on $P^{\prime}$, and $l<0$. By Lemma 5.1 and Lemma 3.5, there is $h \in C^{\infty}\left(\right.$ Aut $P$ ) such that $[h A]$ lies in $\mathscr{M}_{Y}(l, \eta, g)$. So we have $l=l_{Y}, a=0$. We choose $t_{0} \in N$ so that

$$
\int_{\tau-1\left(\left[t_{0}, \infty\right)\right)}\left|F_{A}\right|^{2}<\varepsilon
$$

where we choose $\varepsilon>0$ by Lemma 5.1. Since

$$
\lim _{n \rightarrow \infty} \int_{\tau^{-1}\left(\left[t_{0}, \infty\right)\right)}\left|F_{A_{n}}\right|^{2}=\int_{\tau^{-1}\left(\left[t_{0}, \infty\right)\right)}\left|F_{A}\right|^{2},
$$

we have

$$
\int_{\tau-1\left(\left[t_{0}, \infty\right)\right)}\left|F_{A_{n}}\right|^{2}<\varepsilon \quad\left(n>n_{0}\right)
$$

for some $n_{0} \in N$. Then there exists $h_{n} \in C^{\infty}\left(\operatorname{Aut}\left(Q \times\left[t_{0}, \infty\right)\right)\right)$ such that

$$
\sup _{\tau_{-1}(\ldots \ldots . . .}\left\{\sum_{l=0}^{m}\left|\nabla_{\pi * T}^{(l)}\left(h_{n} A_{n}-\pi^{*} \Gamma\right)\right|^{2}\right\} \leqq \rho e^{-2 \lambda\left(t-t_{0}\right)}
$$

for $t \geqq t_{0}$. Now we apply Ascoli-Arzela's theorem with diagonal argument to deduce that $h_{n} A_{n}$ converges to an ASD connection $A^{\prime}$ in $C^{m-1}$ over compact sets in $\tau^{-1}\left(\left[t_{0},{ }^{\text {TO }}\right)\right)$ So

$$
\sup _{\tau^{-1}([t-1, t+1])}\left\{\sum_{l=0}^{m-1}\left|\nabla_{\pi * T}^{(l)}\left(A^{\prime}-\pi^{*} \Gamma\right)\right|^{2}\right\} \leqq \rho e^{-2 \lambda\left(t-t_{0}\right)}
$$

for $t \geqq t_{0}$. By bootstrapping the equation 


$$
d_{A}\left(h_{n} k_{n}^{-1}\right)=h_{n} k_{n}^{-1}\left(k_{n} A_{n}-A\right)-\left(h_{n} A_{n}-A\right) h_{n} k_{n}^{-1},
$$

we see that there is a subsequence $\left\{h_{n} k_{n}^{-1}\right\}$ (now we relabeled) such that $h_{n} k_{n}^{-1}$ converges to some $u$ in $C^{m-1}$ on $\tau^{-1}\left(\left(t_{0}, t_{0}+1\right)\right)$ By Lemma 3.5, if we replace $\left\{h_{n}\right\}$ by $\left\{r h_{n}\right\}$ for some $r \in \mathscr{R}$, we can suppose that $\tilde{u}$ can be extended to $u^{*}$ over $\tau^{-1}\left(\left[0, t_{0}+1\right)\right.$ ) So we can apply the argument of $[7$, Lemma (4.4.5)] (see also Appendix 1) to patch gauge transformations $k_{n}$ and $r h_{n}$ over $\tau^{-1}\left(\left(t_{0}, t_{0}+1\right)\right)$ Then taking a subsequence, we can find $C^{m-1}$-gauge transformations $\left\{u_{n}\right\}$ on $Y$ such that $u_{n}=h_{n}$ on $\tau^{-1}\left(\left[t_{0}+1, \infty\right)\right)$ and $u_{n} A_{n}$ converges to an ASD connection $A^{\prime \prime}$ on $P$ in $C^{m-2}$ on compact subsets of $Y$. If we. choose $m \geqq 5$, then

$$
\left\|u_{n} A_{n}-A^{\prime \prime}\right\|_{L_{3}^{2}, \delta}(Y)-0(n \rightarrow \infty)
$$

and $\left[u_{n} A_{n}\right],\left[A^{\prime \prime}\right] \in \mathscr{M}_{Y}\left(l_{Y}, \eta, g\right)$. Now by Lemma 3.3, $\left\{u_{n}\right\}$ is in $\mathcal{G}_{P}$.

\section{REFERENCES}

[1] M.F. ATIYAH, New invarıants of 3-and 4-dimensional manifolds, Proc. Sympos. Pure Mathematics (The Mathematical heritage of Hermann Weyl) 48 (1988), 285-299.

[2] M.F. ATIYAH, N.J. Hitchen AND 1. M. SINGER, Self-duality in four dimensional Riemannian geometry, Proc. R. Soc. London Ser. A, 362 (1978), 425-461.

[3] S. Demichelis AND M.H. FREEDMRN, Uncountably many exotıc $R^{4}$ in standard 4-space, J. Differ. Geom. 35 (1992), 219-254.

[4] A. DOLD AND H. WHITNEY, Classification of oriented sphere bundles over a 4complex, Annals of Math. 69 (1959), 667-677.

[5] S.K. DONALDSON, The orientation of Yang-Mills moduli spaces and 4-manifold topology, J. Differ. Geom. 26 (1987), 397-428.

[6] S.K. DONALDSON, Polynomial invariants for smooth four manifolds, Topology 29 (1990), 257-315.

[7] S.K. DonaldSon AND P.B. KRONHEIMER, The geometry of four-manifolds, Oxford Mathematical Monographs, Oxford Univ. Press, 1990.

[8] D.S. FREED AND K.K. UHLENBECK, Instantons and four-manifolds, M.S. R.I. Publications, Vol. 1, Springer, 1984.

[9] R. FRIEDMAN AND J.W. MORGAN, On the diffeomorphismtypes of certain algebraic surfaces. I, II, J. Differ. Geom. 27 (1988), 297-398.

[10] R. E. GOMPF AND T. S. MROWKA, Irreducible four manifoldsneed not be complex, preprint (1991).

[11] Y. KAMETANI AND Y. SATO, O-dimensional moduli spaces of stable rank 2 bundles and differentiablestructures on regular elliptic surfaces, preprint (1991).

[12] D. KOTSCHICK, On manifolds homeomorphıc to $\boldsymbol{C} \boldsymbol{P}^{2} \sharp 8 \boldsymbol{C P}^{\mathbf{2}}$, Invent. math. 95 (1989), 591-600.

[13] P.B. KRONHEIMER, Instanton invariants and flat connections on the Kummer surface, Duke. Math. J. 64 (1991), 229-241.

[14] P.B. KRONHEIMER AND T.S. MROWKA, Gauge theory for embedded surface, I, preprint (1991). 
[15] R.B. LockHART AND R. C. MCOWEN, Elliptic differential operators on noncompact manifolds, Ann. Sc. Norm. Super. Pisa. Cl. Sci. Ser IV, Vol. XII, n. 3, 409-447.

[16] G. MAtic, SO(3)-connections and rational homology cobordisms, J. Differ. Geom. 28, 277-307.

[17] B. MOISHEZON, Complex surfaces and connected sums of complex projective planes, Lecture Notes in Math. 603, Springer, 1977.

[18] C. OKONECK AND A. VAN DE VEN, Stable vector bundles and differentiable structures of Barlow's surfaces, Invent. math. 86, 357-370.

[19] N. E. STEENROD, The topology of fibre bundles, Princeton Mathematical Series, Princeton Univ. Press, 1951.

[20] C. H. TAUBES, Gauge theory on asymptotically periodic 4-manifolds, J. Differ. Geom. 19, 363-430.

[21] M. UE, $A$ remark on the simple invariontsfor elliptic surfaces and their exotic structures not coming from complex surfaces, preprint (1991).

[22] K. K. UHLENBECK, Connections with L $L^{p}$-bounds on curvature, Comm. Math. Phys. 83 (1982), 31-42.

DEPARTMENT OF MATHEMATICS, TOKYO METROPOLITAN UNIVERSITY, MINAMI-OHSAWA $1-1$,

HACHIOJI-SHI, TOKYO 192-03, JAPAN 